\title{
Reverberation in Tidal Disruption Events: Dust Echoes, Coronal Emission Lines, Multi-wavelength Cross-correlations, and QPOs
}

\author{
Sjoert van Velzen ${ }^{1,2} \cdot$ Dheeraj R. Pasham ${ }^{3} \cdot$ Stefanie Komossa ${ }^{4} \cdot{\text { Lin } \text { Yan }^{5} \cdot \text { Erin A. Kara }}^{6}$
}

Received: 13 June 2020 / Accepted: 2 June 2021 / Published online: 1 July 2021

(c) The Author(s) 2021

\begin{abstract}
Stellar tidal disruption events (TDEs) are typically discovered by transient emission due to accretion or shocks of the stellar debris. Yet this luminous flare can be reprocessed by gas or dust that inhabits a galactic nucleus, resulting in multiple reverberation signals. $\mathrm{Nu}-$ clear dust heated by the TDE will lead to an echo at infrared wavelengths $(1-10 \mu \mathrm{m})$ and transient coronal lines in optical spectra of TDEs trace reverberation by gas that orbits the black hole. Both of these signal have been detected, here we review this rapidly developing field. We also review the results that have been extracted from TDEs with high-quality $\mathrm{X}$-ray light curves: quasi periodic oscillations (QPOs), reverberation lags of fluorescence lines, and cross-correlations with emission at other wavelengths. The observational techniques that are covered in this review probe the emission from TDEs over a wide range of scales: from $\sim 1$ light year to the innermost parts of the newly formed accretion disk. They provide insights into important properties of TDEs such as their bolometric output and the geometry of the accretion flow. While reverberation signals are not detected for every TDE, we anticipate they will become more commonplace when the next generation of X-ray and infrared instruments become operational.
\end{abstract}

The Tidal Disruption of Stars by Massive Black Holes

Edited by Peter G. Jonker, Sterl Phinney, Elena Maria Rossi, Sjoert van Velzen, Iair Arcavi and Maurizio Falanga

S. van Velzen

sjoert@strw.leidenuniv.nl

1 Center for Cosmology and Particle Physics, New York University, New York, NY 10003, USA

2 Leiden Observatory, Leiden University, Postbus 9513, 2300 RA Leiden, The Netherlands

3 IT Kavli Institute for Astrophysics and Space Research, Cambridge, MA 02139, USA

4 Max-Planck-Institut für Radioastronomie, Auf dem Hügel 69, 53121 Bonn, Germany

5 Caltech Optical Observatories, Cahill Center for Astronomy and Astrophysics, California Institute of Technology, Pasadena, CA 91125, USA

6 Kavli Institute for Astrophysics and Space Research, Massachusetts Institute of Technology, 77 Massachusetts Avenue, Cambridge, MA 02139, USA 


\section{Introduction}

At the time of writing, the field of tidal disruption event (TDE) research finds itself in an interesting position: most of the new events are found in optical surveys, see the Optical Chapter (van Velzen et al. 2020) of this journal, yet the origin of optical emission from TDEs is unknown. As reviewed in the Emission Mechanisms Chapter (Roth et al. 2020) of this journal, the emission could be powered by an inner accretion disk or by shocks of the intersecting debris streams. A related open question concerns the observed fluence, which is a few orders of magnitude lower than the expected energy released when a star gets disrupted and accreted by a massive black hole (Piran et al. 2015).

The most recent models for the optical emission from TDEs all have been tailored to explain the current set of optical/X-ray properties of TDEs (e.g., peak luminosity, light curve shape, emission line widths). As such, finding new TDEs alone is not sufficient to discriminate between these models. To make progress towards understanding the emission mechanism of TDEs, probing a broader set of observables is key.

This brings us to the topic of this chapter: we will review a number of observational methods that probe beyond a single-wavelength light curve or spectrum. Each of these methods has provided valuable information about the TDE emission mechanism. However, given the requirements of high signal-to-noise or long monitoring campaigns, these observables have been measured for only a handful of events.

The techniques discussed here span a very wide range of physical scales. We start with the largest scale of $\sim 1$ light year, as probed by echoes of the TDE emission from dust and gas, measured using infrared imaging (Sect. 2) and coronal lines (Sect. 3), respectively. Next, we consider correlations between the emission at difference wavelengths that have been reported for the TDE ASASSN-14li (Holoien et al. 2016): a cross-correlation between the X-ray emission and radio emission (Sect. 4.2), which implies a coupling on scales of $\sim 10^{16} \mathrm{~cm}$, and a correlation between the X-ray light curve and the UV light curves (Sect. 4.3), probing scales of $\sim 10^{14} \mathrm{~cm}$. Finally, we consider two results from X-ray timing analysis that probes the innermost parts of the newly formed TDE accretion disks: the detection of quasi period oscillations (QPOs) for some TDEs (Sect. 5) and relativistic reverberation from the jetted TDE Swift J1644+57 (Sect. 6). We close with a brief discussion (Sect. 7).

\section{Infrared Imaging: Dust Echoes}

The reprocessing of TDE light by dust can yield an infrared (IR) echo that probes the environment of TDEs on a scale of $\sim 0.1 \mathrm{pc}$. This subject has seen a flurry of activity in recent years. The possibility of dust reprocessing was explored for the first time by Komossa et al. (2009), who presented a Spitzer observation (Fig. 1) of the coronal-line selected TDE SDSS J0952+2143 (events selected by coronal lines are discussed in Sect. 3). The important theoretical paper on dust echoes by $\mathrm{Lu}$ et al. (2016) made a prediction that infrared emission from a few on-going TDEs could be detectable with imaging observations. Indeed a few months later, three papers that presented observations of dust echoes for different TDEs all appeared in the same week (van Velzen et al. 2016; Jiang et al. 2016; Dou et al. 2016) —although, as evidenced by van Velzen et al. (2015), some of these echoes were detected and studied prior to the forecast of Lu et al. (2016).

In the following three subsections we will review these advances in more detail. First, we give a brief overview of the theoretical background of dust echoes. Next, we present the observations of dust echoes using follow-up observations of TDEs. And third, we look at the possibility to discover new TDEs using IR observations of echoes. 


\subsection{Theory of Dust Reverberation}

Reverberation by dust has long been used as a tool to study the dust around active galactic nuclei (AGN) (e.g., Barvainis 1987) and supernovae and supernovae remnants (e.g., Dwek 1983). The concept is relatively simple. Dust will efficiently absorb optical/UV/X-ray photons, this process heats the dust to a maximum temperature, which yields isotropic emission that peaks at a few micron. The time delay between the fluctuations in emission from the accretion disk and the IR emission yields the inner radius of the dust region (for an example of the application of this technique in AGN, see Koshida et al. 2014). In a TDE, the black hole was not actively accreting, which will modify our expectation of the dust reprocessing signal. The lack of accretion disk emission in the years prior to the TDE means the dust can survive closer to the black hole. But at the same time, the lack of gas supply to the galaxy center implies we could expect a very low dust covering factor and thus a weak reverberation signal compared to active galaxies.

To estimate relevant timescale of a dust echo we have to equate the absorbed flux at radius $R$ from the black hole to the emission by the dust particles. The latter is relatively well-constrained, dust absorption is efficient across the wavelength range where TDEs emit most of their energy. However the dust emission is more uncertain because it depends on the model for the dust particles, which can include grain composition, size, and the shape of the emission spectrum as free parameters.

Fortunately, the uncertainty on the grain properties is reduced by the extreme conditions of a TDE, which reduce the type of dust that we expect to be visible. The grains that can survive closest to the black hole dominate the IR echoes because they radiate the received energy in the shortest amount of time. Furthermore, if the dust emission efficiency scales with the square of the dust size (Draine and Lee 1984), we expect the largest grains to dominate the reprocessing emission. Hence most authors have normalized their predictions for TDE dust emission to a single grain size, using $a \approx 0.1 \mu \mathrm{m}$, the typical maximum dust size for the Milky Way (Weingartner and Draine 2001).

After restricting to "graphite-like" dust particles, because these have the highest sublimation temperature and should thus dominate the reprocessing signal, van Velzen et al. (2016) arrive at the following expression for the sublimation radius

$$
R_{\text {sub }}=0.15\left(\frac{L_{45}}{a_{0.1}^{2} T_{1850}^{5.8}}\right)^{1 / 2} \mathrm{pc} .
$$

Here $L_{45}$ is the bolometric luminosity of the TDE in units of $10^{45} \mathrm{erg} \mathrm{s}^{-1}, a_{0.1}$ is the grain size in units of $0.1 \mu \mathrm{m}$, and $T_{1850}$ is the temperature of the dust, normalized to the expected sublimation temperature of $1850 \mathrm{~K}$. Equation (1) is derived with the common assumption that dust absorption is $100 \%$ efficient across the relevant wavelength range $\left(Q_{\mathrm{UV}}=1\right)$. If we use a dust model that approximates a two-component medium consisting of both graphite and silicate grains (Waxman and Draine 2000; Lu et al. 2016) the sublimation radius is

$$
R_{\text {sub }}=0.12\left(\frac{L_{45}\left(1+0.1 a_{0.1} T_{2300}\right)}{a_{0.1} T_{2300}^{5}}\right)^{1 / 2} \mathrm{pc},
$$

with $T_{2300}$ the dust temperature normalized to $2300 \mathrm{~K}$.

From both Eq. (1) and Eq. (2) we find a typical size scale of $0.1 \mathrm{pc}$ for the emitting region, which implies a timescale of a few months for the reprocessing signal. Because this timescale can be measured from the IR light curve (Fig. 2), solving $R_{\text {sub }}$ for $L_{45}$ yields an 


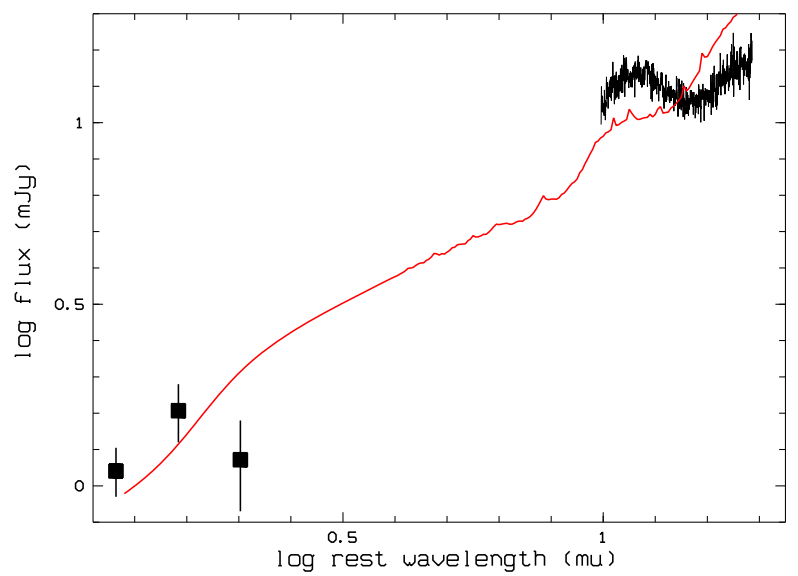

Fig. 1 IR SED of SDSS J0952+2143 observed with Spitzer (black solid line) and 2MASS (pre-flare; filled squares), reproduced from Komossa et al. (2009). The plot emphasizes the large Spitzer IR luminosity at outburst in comparison to pre-outburst NIR data, and the characteristic Spitzer spectral shape, measured for the first time in a TDE. Overplotted is the mean Spitzer IR SED of accreting extragalactic sources (solid red line; Netzer et al. 2007). Note a conversion error in Komossa et al. (2009) when plotting that comparison SED (their Figs. 7 and 8), which led to an incorrect presentation of the shape of that SED, corrected by Komossa (2010)

estimate of the bolometric luminosity of the TDE. This approach only works if the observed IR emission is dominated by a single shell that emits at the sublimation temperature. If the dust echo contains emission from larger scales (e.g., because the galaxy is devoid of dust at $R<R_{\text {sub }}$ ), a measurement of the dust temperature is also needed to estimate $L_{\text {bol }}$ (e.g., Kool et al. 2020).

While the bolometric luminosity is estimated from the shape of the reprocessing light curve, the covering factor $\left(\Omega_{d}\right)$ follows from the amplitude of the echo. We define $\Omega_{d} \equiv$ $E_{\text {bol }} / E_{\text {dust }}$, with $E_{\text {dust }}$ the total energy radiated by the dust and $E_{\text {bol }}$ the total radiated energy of the TDE (integrated over the wavelength where dust absorption is efficient).

\subsection{Infrared Echoes Detected in Follow-up Observations}

The first TDE with IR follow-up observations was SDSS J0952+2143 (Komossa et al. 2008, 2009), which was selected based on variable coronal line emission and variable optical, NIR and UV continuum emission (this class of events is discussed in full detail in the next section). This source was observed once with the Spitzer IRS spectrograph at 10-20 $\mu \mathrm{m}$ (Fig. 1), yielding three main results (Komossa et al. 2009). First, the detection of a large MIR luminosity of $L_{10-20} \mu \mathrm{m}=3.5 \times 10^{43} \mathrm{erg} \mathrm{s}^{-1}$ in the Spitzer band which implies a large $\mathrm{X}$-ray luminosity (not observed directly) and efficient reprocessing. Second, a blackbody fit to the IR spectrum gave an estimate of the radius of the IR emission site $r \approx 0.5 \mathrm{pc}$. Third, the characteristic emission features from PAHs and silicate dust were detected in the spectrum.

The single Spitzer observation by Komossa et al. (2009) was carried out about 4 years after the start of the optical flare (Palaversa et al. 2016). The transient nature of the IR at emission was confirmed later via WISE observations at $12 \mu \mathrm{m}$ (the WISE W3 band), obtained 1.9 year after the Spitzer spectrum, showing that the flux at this wavelength has decreased by a factor of 1.8 (Dou et al. 2016). 
Table 1 Events with transient infrared emission

\begin{tabular}{llll}
\hline Name & Host galaxy type & $\begin{array}{l}\text { Peak IR luminosity* } \\
\left(\mathrm{erg} \mathrm{s}^{-1}\right)\end{array}$ & Reference \\
\hline PTF-09ge & Quiescent & $10^{41.8}$ & 1 \\
PTF-09axc & Quiescent (E+A) & $10^{42.6}$ & 1 \\
ASASSN-14li & Quiescent (E+A) & $10^{41.6}$ & 2 \\
AT2019dsg & Quiescent & $10^{43.0}$ & $3^{\dagger}$ \\
NGC 5092 & Quiescent & $10^{42.6}$ & 4 \\
IRAS F01004-2237 & ULIRG & $10^{44.3}$ & 5 \\
PS16dtm & AGN (Type 1) & $10^{43.0}$ & 6 \\
PS1-10adi & AGN (Type 1) & $10^{44.0}$ & 7,8 \\
SDSS J1657+2345 & AGN (Type 2) & $10^{43.9}$ & 9 \\
SDSS J0227-0420 & AGN (Type 1) & $10^{43.6}$ & 10 \\
Arp 299-B AT1 & LIRG & $10^{43.8}$ & 11 \\
AT2017gbl & LIRG & $10^{43.2}$ & 12 \\
MCG-02-04-026 & AGN (Type 1) & $10^{43.2}$ & 13 \\
SDSS J0952+2143 & Quiescent (HII-type) & $10^{43.9}$ & 14,15 \\
\hline
\end{tabular}

Notes - This table includes events published up to 2020, for more recent IR-detection from optical TDEs see Jiang et al. (2021), which appeared while this article was under review

*Peak observed luminosity $\left(v L_{v}\right)$ based on host-subtracted WISE data (except for SDSS J0952+2143 the peak flux of which is based on Spitzer photometry)

${ }^{\dagger}$ The peak IR luminosity of AT2019dsg is based the host-subtracted neoWISE magnitude of $W 1=13.1$ (Vega)

References: 1 (van Velzen et al. 2016), 2 (Jiang et al. 2016), 3 (Stein et al. 2020), 4 (Li et al. 2020), 5 (Dou et al. 2017), 6 (Jiang et al. 2017), 7 (Kankare et al. 2017), 8 (Jiang et al. 2019), 9 (Yang et al. 2019), 10 (Liu et al. 2020), 11 (Mattila et al. 2018), 12 (Kool et al. 2020), 13 (Sun et al. 2020) 14 (Komossa et al. 2009), 15 (Dou et al. 2016)

Infrared photometric data of optically discovered TDEs are often obtained from the Wide-field Infrared Space Explorer (WISE; Wright et al. 2010) which has been systematically scanning the whole sky in 3.4 and $4.6 \mu \mathrm{m}$ since 2010 . The primary (cryogenic, 4 band) and post-cryogenic WISE surveys ran from Jan 2010 to Feb 2011. Due to lack of funding, the WISE space telescope was placed in hibernation between 2011 to 2013. On December 13, 2013, NASA reactivated the WISE telescope and started an all sky survey called Near-Earth Object WISE Reactivation mission (NEOWSIE-R; Mainzer et al. 2014). NEOWISE-R is still operating since December 2013. It scans the most part of the sky at 3.4 and $4.6 \mu \mathrm{m}$ every half year, with $10-20$ single exposures in each visit.

Based on the WISE archive photometry, several studies have examined the mid-IR (MIR) light curves (LC) of TDEs identified in optical imaging surveys with spectroscopic followup observations, see the Optical Chapter (van Velzen et al. 2020) of this journal. These include ASASSAN-14li (Jiang et al. 2016), PTF09ge, and PTF09axc (van Velzen et al. 2016). Several optical flares in active galaxies that have been interpreted as TDEs are also found to show mid-IR flares, see Table 1. Dou et al. (2016) presented WISE detections of four SDSS galaxies with variable coronal emission lines (see Sect. 3 of this chapter). It is interesting to note that the MIR LCs of the spectroscopically-selected TDEs, if detected in the first place, all have small amplitude variations with $\Delta m \sim 0.1$ magnitude. For ASASSN14li and PTF-09ge, the peak MIR LC corresponds to infrared luminosity $L_{\mathrm{IR}} \sim 10^{41} \mathrm{erg} \mathrm{s}^{-1}$ 


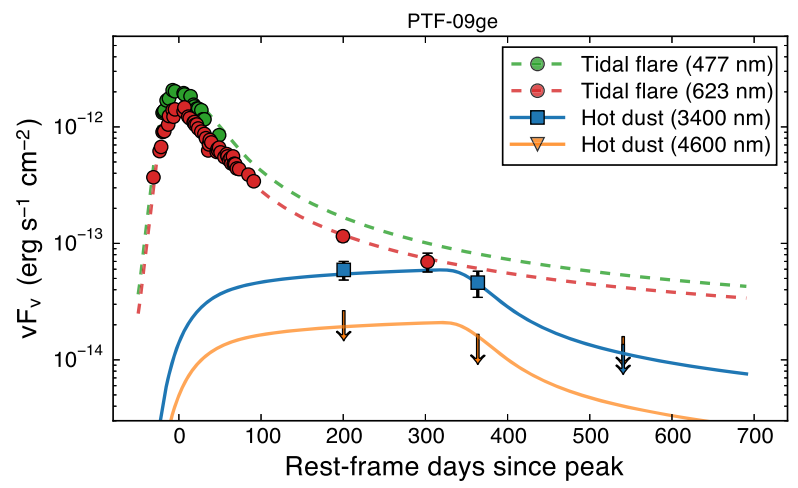

Fig. 2 The optical (Arcavi et al. 2014) and infrared (van Velzen et al. 2016) light curve of the TDE PTF-09ge. The solid lines show the dust reverberation light curve, which is derived from the optical light curve using only the radius of the dust shell and the covering factor as free parameters. The former sets the duration of the event, while the latter affects only the overall normalization. The best-fit parameters are $R=0.15 \mathrm{pc}$ and $f_{\text {dust }}=0.01$. Figure reproduced from van Velzen et al. (2016)

and $L_{\mathrm{IR}} \sim 10^{42} \mathrm{erg} \mathrm{s}^{-1}$, respectively. As shown in Table 1, this luminosity is between one and two an orders of magnitude lower than the peak IR luminosity of the TDE candidates in active galaxies.

The peak phases of the MIR LCs display time delays relative to the optical LCs, with $\Delta t \sim 100-200$ days. This time delay translates to the radius of dust medium of about $0.1 \mathrm{pc}$, somewhat larger than the dust sublimation radius of $0.04 \mathrm{pc}$ as derived from $L_{U V}$. In general, these numbers are consistent within the context of MIR LCs as the result of the dust echo of the UV/optical flares. Three additional parameters - the covering factor, dust mass and geometry, and the flare bolometric luminosity (via Eq. (1)) - can be estimated from the MIR LCs, although with considerable uncertainty because dust temperatures are not well constraint with only one color ([3.4 - 4.6] $\mu \mathrm{m})$. For PTF-09ge (Fig. 2), van Velzen et al. (2016) marginalized over the uncertainty on the dust temperature and included the measurement uncertainty on $R_{\text {sub }}$ to find $\log _{10}\left(L_{\text {bol }}\right)=44.9_{-0.6}^{+0.1}$ and $\log _{10} \Omega_{d}=-2.0 \pm 0.2$.

Better spectroscopic coverage is one area of improvement for future near-IR follow-up observations. And with well-sampled IR light curves, we can obtain a better handle of the geometry of the dust, allowing us to differentiate between a torus model (Dou et al. 2016; Sun et al. 2020) and a polar or spherical dust geometry (van Velzen et al. 2016; Mattila et al. 2018; Kool et al. 2020).

The lower average IR luminosity of TDEs in quiescent galaxies compared to events selected by their variable coronal emission lines (ECLEs, see Sect. 3) suggests that the covering factor and the dust mass that participates in the reprocessing is larger for the ECLEs. Intriguingly, the first WISE detections of the MIR flare in SDSS J0952+214 is 3-5 years after the changes of coronal emission lines. Together with the high IR luminosity, this long time scale of the variation could be an indication that these do not belong to the same class as the TDEs selected based on optical photometric and follow-up spectroscopy. We discuss the ambiguity between changing-look AGNs and TDEs, and the possible ways to separate them in Sect. 2.3 below.

Infrared echo offers a unique tool to probe the dusty circumnuclear medium at close distances to the central supermassive black holes. One interesting example is PS16dtm, which could be a TDE in a Narrow Line Seyfert 1 galaxy (Blanchard et al. 2017) — however see Moriya et al. (2017) for a different interpretation. The MIR LC of PS16dtm appears to rise 
about 11 days before the first epoch of optical detection (Jiang et al. 2017). This can be explained by a pre-existing dusty medium with a size of 11 light days $\left(2.8 \times 10^{16} \mathrm{~cm}\right)$, with a sufficient high covering factor, blocking the initial optical/UV light. This size is similar to the dust sublimation radius inferred from the quiescent AGN with $L_{\mathrm{bol}} \approx 5 \times 10^{42} \mathrm{erg} \mathrm{s}^{-1}$. After the TDE flare, the high flux of UV/X-ray photons destroys the dust grains and enlarges the inner radius of the dust free cavity to $\sim 70$ light days $\left(2 \times 10^{17} \mathrm{~cm}\right)$. Additional Fe elements previously locked up by the dust grains are now released into the ionized gas medium, potentially explaining the abundant FeII emission lines observed in the follow-up spectra by Blanchard et al. (2017).

Infrared echoes appear to be ubiquitous to the class of highly energetic transients from the centers of active galaxies (Kankare et al. 2017). An example is PS1-10adi (Kankare et al. 2017), which has a very high MIR luminosity of $\sim 10^{44} \mathrm{erg} \mathrm{s}^{-1}$ (Jiang et al. 2019).

Infrared flares interpreted as dust reprocessing signals of energetic transients have also been found in star-forming and dusty luminous infrared galaxies (LIRGs; the infrared emission in these galaxies is thought to be mainly powered by star formation Genzel and Cesarsky 2000). The best-studied case is Arp 299-B AT1, which was observed with high spatial resolution radio observations combined with near- and mid-IR observations covering a period of over 10 years (Mattila et al. 2018). Based on this excellent data, a TDE is the preferred explanation proposed by Mattila et al. (2018).

Due to the small sample size of LIRGs that have been monitored for IR flares, this interpretation would imply an elevated rate of TDEs for this subset of galaxies. Likewise, a TDE interpretation of the transients found by Tadhunter et al. (2017) in a small sample of ultra-luminous infrared galaxies (ULIRGs) would imply a factor $\sim 100$ enhancement to the TDE rate for this galaxy class (Stone et al. 2018).

\subsection{Infrared Flares from Quiescent and Active Galaxies: A Tool to Find Dust-Obscured TDEs?}

TDE studies using optical all-sky transient surveys face several important questions. First, tidal disruption of stars by central black-holes can in principle also occur in galaxies with active galactic nuclei (AGN) and dusty infrared luminous star-forming galaxies. Second, how do we distinguish Changing-Look (CL) AGNs from TDEs? While the underlying physics has similarities, these types of transients may probe different scales (time, size and mass) of accretion phenomena.

Some TDE candidates have been found in Seyferts and dusty LIRG/ULIRGs, as listed in see Table 1. These studies confront a common question-could these flares be due to AGN changing their states, i.e., CL AGNs?

The observational ambiguities between CL AGNs and TDEs are abundant-see the Imposters Chapter (Zabludoff et al. 2021) of this journal. Separation often requires multiwavelength data over a long time scale of several years, as has been obtained for the systems discussed in Sect. 3. Particularly, the properties of a "turn-on" AGNs, transitioning from a quiescent to an active accreting state, can be easily confused with that of a TDE, as shown by Yan et al. (2019). Using an extensive dataset of Swift UV, optical, mid-IR LCs and spectroscopy over 1200 days, this study made comprehensive comparisons between CL AGN, TDE and unusual SN IIn and showed that the flare in SDSS1115+0544 is a rapid "turn-on" of a type 1 AGN, changing from its earlier quiescent state. Yan et al. (2019) highlighted that having only LCs or variable spectra, including high ionization coronal lines, is not sufficient to make conclusive classifications (see also the detailed discussion by Komossa et al. 2008). A potential solution could be collecting larger samples of the various classes of TDEs, including events in AGNs and LIRGs/ULIRGs. 
Potentially, using infrared variability should offer an effective means to identify TDE candidates in dusty galaxies. Such systematic searches using WISE mid-IR time series photometry are being carried out and below we discuss an example of this approach.

Using only the WISE data between December 2009 and February 2011, Wang et al. (2018) searched for potential TDE candidates among SDSS galaxies with spectra which present no indications of AGN. Only based on their mid-IR LCs, they found a total of 14 TDE candidates from quiescent SDSS galaxies at $z<0.22$ with estimated blackhole masses of $10^{7-8} M_{\odot}$. The estimated rate is $\sim 10^{-4}$ galaxy $^{-1} \mathrm{yr}^{-1}$ with large uncertainties. As concluded by these authors, these 14 candidates could contain a good fraction of CL AGNs which show short time scale episodic variability.

The second ongoing search is done by the same group but with the entire WISE data between December 2009 and 2018 (see Yan et al. 2019 for a brief description of the full survey). The systematic search for infrared flares was done among all SDSS spectroscopic confirmed galaxies at $z<0.35$. A total of 137 galaxies were selected with flare amplitudes $\geq 0.5$ magnitude in at least one WISE band (Jiang et al. 2020). This sample does not have complete spectroscopic classification. It contains a mixture of various types, including a small fraction of supernovae and radio loud AGNs. Jiang et al. (2020) speculates that a large fraction of this sample are possible dust echoes of TDEs and CL AGNs. One example with extensive spectroscopy is SDSS1115+0544, a rapid "turn-on" type-1 AGN (Yan et al. 2019).

Radio follow-up observations of 16 WISE-selected flares by Dai et al. (2020) have yielded a high detected fraction of $75 \%$ (for a typical $5 \sigma$ upper limit $60 \mu \mathrm{Jy}$ at $5.5 \mathrm{GHz}$ ). Based on pre-flare SDSS spectroscopy, about half of targets in this sample can be classified as AGN, while most of the non-AGN show evidence for active star formation. A radio detection rate of $75 \%$ is much higher than the fraction of AGN or TDEs that are considered to be "radio-loud", see the Radio Chapter (Alexander et al. 2020) of this journal. Based on this high detection rate and similarities with other radio-detected IR flares (Mattila et al. 2018; Kool et al. 2020), one could conclude that IR-selection yields a subclass of TDEs that are often accompanied by powerful jets, with total energies of $\sim 10^{52} \mathrm{erg}$. Such powerful jetted TDEs have been observed before (see Bloom et al. 2011; Zauderer et al. 2011, and the Gamma-ray Chapter of this book), although the estimated volumetric rate of these events, $\sim 1 \mathrm{Gpc}^{-1} \mathrm{yr}^{-1}$ or $\sim 10^{-7}$ galaxy $^{-1} \mathrm{yr}^{-1}$ (van Velzen et al. 2018), is three orders of magnitude lower than typical rate of WISE-selected flares from galaxies with SDSS spectra $\left(\sim 10^{-4}\right.$ galaxy $^{-1} \mathrm{yr}^{-1}$; Wang et al. 2018). Alternatively, one could conclude that a selection of IR flares in WISE data yields a sample of active black holes in a special phase of their evolution, during which a large change in the accretion rate triggers or rekindles jet activity, leading to a higher detection rate of radio emission.

\section{Optical Spectroscopy: Emission-Line Echoes}

\subsection{Introduction}

If TDEs occur in a gas-rich environment, their luminous electromagnetic radiation will photoionize any surrounding gas and will be reprocessed into emission lines. These are then very useful probes of the physical conditions and kinematics of the gaseous material in the SMBH vicinity, including the disrupted star itself.

Photoionization by the luminous TDE radiation, which often peaks in the EUV or soft X-rays, will then not only produce Hydrogen Balmer lines, but in particular also highionization lines like HeII $\lambda 4686$ and coronal lines. The ions which produce these lines have 
Table 2 Extreme coronal line emitters

\begin{tabular}{lll}
\hline Full (short) name & Redshift & First reference \\
\hline SDSS J095209.56+214313.3 (J0952+2143) & 0.0789 & Komossa et al. 2008 \\
SDSS J074820.67+471214.3 (J0748+4712) & 0.0615 & Wang et al. 2011 \\
SDSS J134244.42+053056.1 (J1342+0530) & 0.0366 & Wang et al. 2012 \\
SDSS J135001.49+291609.7 (J1350+2916) & 0.0777 & Wang et al. 2012 \\
\hline
\end{tabular}

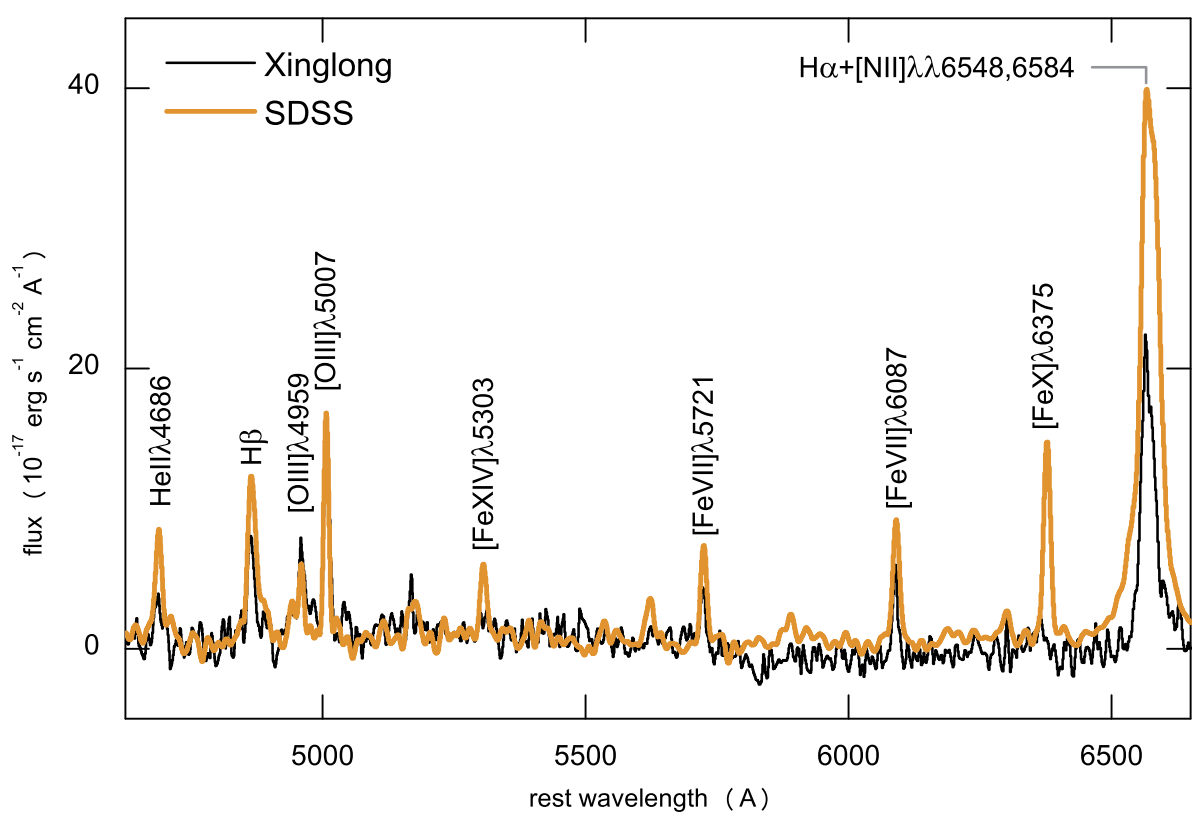

Fig. 3 Optical SDSS spectrum of SDSS J0952+2143 during the high-state (orange) from 2005 with several strong iron coronal lines and other transitions marked. A zoom on the H $\alpha$ profile (shown above in degraded spectral resolution, to match the same resolution as the Xinglong spectrum) is presented in Fig. 4 in high resolution. The first optical follow-up spectrum from 2007 (taken with the Xinglong telescope, plotted in black) revealed the strong fading of the highest-ionization lines (Komossa et al. 2008)

their ionization potentials (IPs) in the EUV to soft X-ray domain. Several such events have been identified in the last decade based on Sloan Digital Sky Survey (SDSS) spectroscopy and multi-wavelength follow-up observations (Komossa et al. 2008, 2009; Wang et al. 2011, 2012; Yang et al. 2013; Palaversa et al. 2016; Dou et al. 2016) and these are discussed below.

The best-studied of these events, SDSS J0952+2143, (Table 2) was first noticed because of its unusual Balmer and HeII emission profiles and especially the exceptional strength of its high-ionization iron lines relative to [OIII] $\lambda 5007$ (Komossa et al. 2008) which were, however, not permanent, but faded away dramatically on a timescale of years (Komossa et al. 2009; Yang et al. 2013), see Fig. 3. A systematic search in SDSS for similar events (Wang et al. 2012) let to the identification of six more, of which three (Yang et al. 2013) showed strongly fading coronal lines (Table 2). Key selection criterion was the presence of bright coronal line emission of [FeX]-[FeXIV] in SDSS spectra, which had dropped significantly in intensity in dedicated follow-up optical spectroscopy. 
Among these extreme coronal line emitters (ECLEs hereafter), SDSS J0952+2143 at redshift $z=0.079$ is the one with the most luminous coronal lines, and the densest multiwavelength coverage both near the high-state and in subsequent years (Komossa et al. 2008, 2009) to which Palaversa et al. (2016) added a long-term optical light curve. Follow-up spectroscopy three years after the SDSS high-state spectrum revealed that the Balmer and coronal lines had strongly declined (Fig. 3). The fact that the outburst extended into the high-energy regime was indirectly inferred from the presence of the luminous, fading coronal lines, while at lower energies continuum variability was directly detected in the NIR, optical and UV (Komossa et al. 2008), followed by a continuous fading of the long-term optical light curve (Palaversa et al. 2016).

The host galaxy of SDSS J0952+2143 was classified as inactive, as no signs of permanent AGN activity were identified; based on line-ratios, NIR colours, and faint X-rays in low-state (Komossa et al. 2008, 2009; our Sect. 3.5). Therefore, the observations are consistent with a TDE which happened in a gas-rich environment.

In addition to the ECLs, fading broad Balmer and He line emission dominate the optical spectrum, with asymmetric profiles, further covered in the Optical Chapter (van Velzen et al. 2020) of this book.

\subsection{Iron Coronal Line Diagnostics}

The presence of the luminous and transient high-ionization lines in optical spectra of the galaxies immediately implied that the sources underwent luminous electromagnetic flares which extended into the soft X-ray regime - not directly observed at all wavebands, but indirectly inferred from the presence of the fading emission lines, as the ions which produce these lines have their ionization potentials (IPs) in the EUV to soft X-ray regime. The iron lines, with initial luminosities up to $L_{[\mathrm{FeVII}]-[\mathrm{FeXIV}]}>10^{40} \mathrm{erg} \mathrm{s}^{-1}$ in SDSS J0952+2143, faded away on the timescale of years, and the degree of ionization changed from higher ([FeX] to [FeXIV]) to lower ([FeVII]), and up to the complete lack of coronal lines 5-8 years after high-state.

Further, the iron lines are known to be sensitive diagnostics of the physical conditions in the line emitting gas. Individual line ratios enable us to distinguish whether the main ionization mechanism is collisional ionization or photoionization, while other line ratios are density and temperature sensitive (e.g., Nussbaumer et al. 1982; Keenan and Norrington 1987; Dere et al. 2009). Based on these diagnostics, gas temperatures of the order of (1.5 $3) \times 10^{4} \mathrm{~K}$ were inferred, consistent with a photoionization origin of the emission lines, and gas densities around $n=10^{7} \mathrm{~cm}^{-3}$ (Komossa et al. 2009; Wang et al. 2012).

\subsection{Oxygen Line Diagnostics}

In SDSS J0952+2143, the intensity ratio of [OIII]4363/[OIII]5007 is exceptionally high (Komossa et al. 2008). [OIII]4363/[OIII]5007 $\simeq 0.2-0.3$ is significantly above the value observed in AGN and above photoionization predictions for a large parameter range (Komossa and Schulz 1997; Groves et al. 2004). The observed value implies a high-density regime, where the ratio [OIII]4363/[OIII]5007 no longer only depends on temperature, but becomes a density diagnostic (Osterbrock 1989; Dopita and Sutherland 2003). The observed value implies $n=10^{7} \mathrm{~cm}^{-3}$ (for $T=(2-5) \times 10^{4} \mathrm{~K}$ ), and the [OIII]4363 emission therefore arises in a similar region as the ECL emission (Komossa et al. 2009).

Two other ECLEs, SDSS J 0748+4712 and SDSS J1342+0530, show an unusual ratio $[\mathrm{OIII}] 5007 /[\mathrm{OIII}] 4959 \approx 2$ in late-time spectra, strongly deviating from the canonical the- 

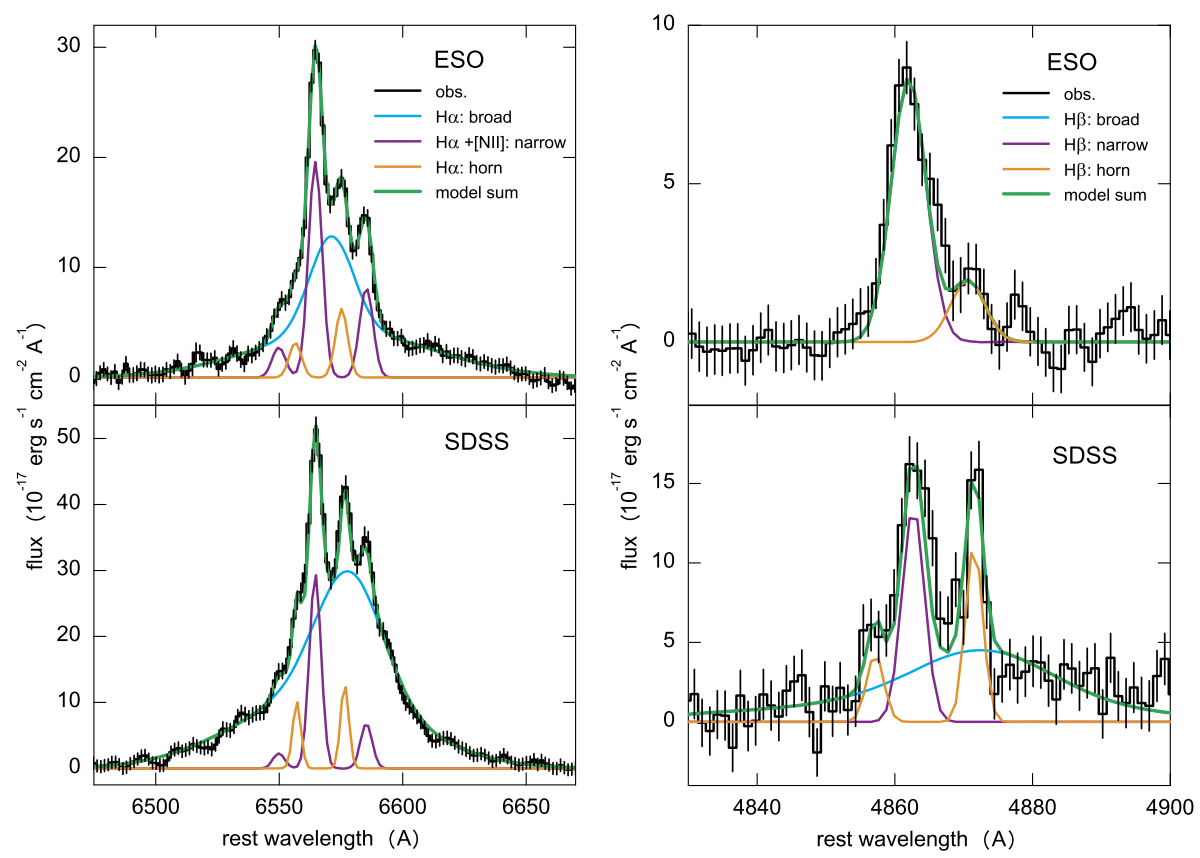

Fig. 4 Hydrogen emission-line profiles of SDSS J0952+2143 ( $\mathrm{H} \alpha$ left, $\mathrm{H} \beta$ right $)$ taken at SDSS high-state in 2005, and with the ESO NTT in 2008. In addition to an asymmetric, broad base (blue), two narrow horns (orange) are clearly present. Their width is unresolved. Adapted from Komossa et al. (2009)

oretical value of 3 observed in all AGN, and interpreted as sign of high density and high optical depth. Unless the metallicity is exceptionally high $(Z>10)$, the data imply that the [OIII] emission region is optically thick to electron scattering (Yang et al. 2013).

\subsection{Balmer-Line Diagnostics: Double-Peaked Horns in $\mathrm{H} \alpha$ and $\mathrm{H} \beta$}

A narrow double-peaked structure in the Balmer lines has been detected in the optical spectra of SDSS J0952+2143 (Komossa et al. 2008), not seen in any other of the ECLEs (Wang et al. 2012), or any other source we are aware of. At high-state, the $\mathrm{H} \alpha$ and $\mathrm{H} \beta$ lines of SDSS J0952+2143 show a multi-component profile, which can be decomposed into at least three distinct components (Fig. 4): (1) a broad component of $\operatorname{FWHM}(\mathrm{H} \alpha)=1930 \mathrm{~km} / \mathrm{s}$, asymmetric and redshifted by $570 \mathrm{~km} / \mathrm{s}$, (2) a narrow component at the same redshift as other narrow forbidden emission lines, and (3) two very narrow horns (FWHM $<200 \mathrm{~km} / \mathrm{s}$, unresolved), one redshifted and the other blueshifted. While line intensities of the Balmer line components and especially the two narrow horns had faded significantly 3 years after the SDSS high-state, their velocity shifts of $v=540 \mathrm{~km} / \mathrm{s}$ (redshifted horn) and $v=-340$ $\mathrm{km} / \mathrm{s}$ (blueshifted horn) remained remarkably constant (Komossa et al. 2009).

The exact nature of the double-horn emission remains unknown at present. The line shape, i.e., the double-peaked profile, could arise in a ring or disk geometry or a two-sided outflow. Surprisingly, the two horns do not have a counterpart in any other of the emission lines. While at first glance, this fact could imply high gas density, several of the strong 


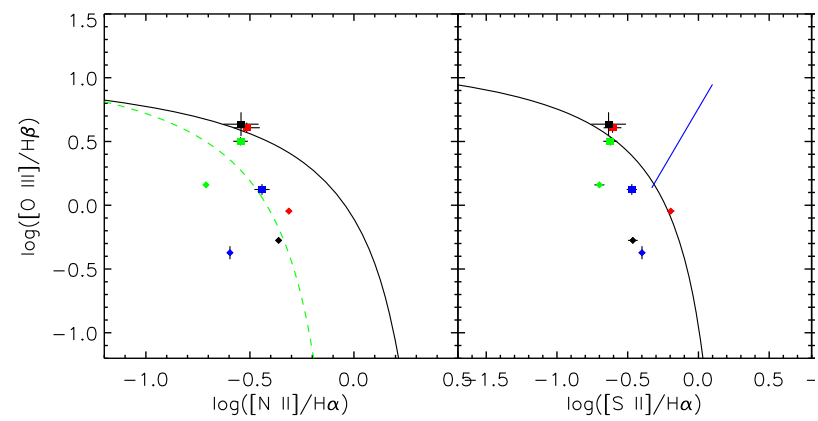

Fig. 5 Location of ECLEs in diagnostic diagrams. They are located in the starburst regime (small symbols), and shift towards higher excitation in the later optical spectra (large squares) which exclude more of the outer starburst emission. The black line marks the dividing line between starformation (lower left) and AGN (upper right), while the blue line represents the deviding line between AGN (above the line) and LINERS (below the line). The region between the green dotted line and black line represents the extreme starburst regime (see Yang et al. (2013))

coronal lines have very high critical density, too, and yet they do not show multiple horns. Komossa et al. (2009) therefore discussed the possibility that these lines form in collisionless shocks. Such shocks produce strong $\mathrm{H}$ lines with a two-component profile composed of a narrow component contributed by cold $\mathrm{H}$ atoms, and a broad component which arises from $\mathrm{H}$ atoms that have undergone charge transfer reactions with hot protons while other optical forbidden lines are very faint (e.g., Raymond et al. 1995; Heng and McCray 2007). Alternatively the broad underlying Balmer line with its two additional narrow horns may represent emission from the inner TDE accretion disk (Komossa et al. 2008).

\subsection{A Note on the Interpretation of These Events}

In summary, four events were observed which showed strong emission-line flaring in response to an optical/UV/X-ray outburst. These ECLEs reside in $\operatorname{sub} L_{*}$ disk galaxies (Wang et al. 2012). A SMBH mass from stellar absorption lines of $7 \times 10^{6} \mathrm{M}_{\odot}$ was estimated in one case (Komossa et al. 2008). Three outburst scenarios have been discussed in the literature.

The first outburst scenario involved very unusual AGN variability. However, no positive evidence for the presence of AGN has been identified: emission-line ratios are in the starburst (HII) regime of diagnostic diagrams (Fig. 5), NIR colours imply quiescent galaxies, and post-flare X-ray emission, only measured in SDSS J0952+2143, is below that of classical AGN $\left(L_{\mathrm{x}}=10^{41} \mathrm{erg} \mathrm{s}^{-1}\right)$ (Komossa et al. 2008, 2009; Wang et al. 2012).

Alternatively, a SN origin has been discussed (Komossa et al. 2009; Wang et al. 2011). However, the very luminous iron coronal lines, especially of SDSS J0952+2143, do imply luminous high-energy emission never observed in SNe and the coronal lines are a factor 100 more luminous than the record holder among SNe, making this event very different from any known type of SN. Further, the longevity of the UV emission, detected 1.8 years after the start of the flare (Palaversa et al. 2016), strongly disfavors an SN origin.

Finally, the events can be interpreted as TDEs in a gas-rich environment, which is preferred explanation by most authors (Komossa et al. 2008, 2009; Wang et al. 2012; Palaversa et al. 2016). In this scenario, the radiation from the temporary accretion disk photoionizes any circumnuclear gaseous material as well as the disrupted star itself. 


\section{Cross Correlations}

\subsection{Introduction and Methodology}

Multi-wavelength photometric reverberation mapping using X-ray, optical, UV, and radio time series of actively accreting SMBHs, i.e., AGN, and stellar-mass black holes have yielded significant insights into the geometries of structures surrounding these systems (e.g., McHardy et al. 2018; Edelson et al. 2015; Marscher et al. 2018; Gandhi et al. 2008; O'Brien et al. 2002; Chatterjee et al. 2009). The underlying method in these studies is to first check for similarity in variability between two light curves derived from two distinct wavebands, and then measure time lags, if any, between them. The hypothesis is that if two light curves from two distinct wavebands vary in the same manner, then they are likely driven by a common physical mechanism.

Under the assumption that the emission from the reference waveband comes from very near to the black hole, any correlations and subsequently time lags give a measure of how far the reverberating regions are with respect to the black hole. The direction (leading vs lagging the emission from near the black hole) and the magnitudes of the time leads/lags vs wavelength essentially give a 1-dimensional map of the geometry of the system under consideration (e.g., Fig. 6 of Cackett et al. 2018). Thus, reverberation mapping analysis provides an invaluable tool to probe spatial scales which cannot be resolved with our current telescopes. For accreting stellar-mass black hole binaries the relevant timescales are between a fraction of a second to a few tens of seconds (e.g., Gandhi et al. 2008; O'Brien et al. 2002) while for AGN they are anywhere between a couple of minutes to a couple of months (e.g., Edelson et al. 2015).

One of the main statistical tools used in reverberation mapping analyses is the so-called cross-correlation function (or CCF). Mathematically, this defines the amount of similarity between two time series as a function of time offset (time lag/lead) between them. CCFs are usually normalized such that a value of unity means a perfect match between the two input time series while a value of negative unity implies they are exactly anti-correlated. A value near zero implies no correlation. A CCF is straightforward to compute when the two time series of interest are uniformly sampled with the same time binning. However, within the context of astronomical time series, often the data are non-uniformly sampled owing to the various observing constraints associated with telescopes. For these unevenly sampled cases, the three most widely used forms of $\mathrm{CCF}$ are the interpolated cross-correlation function (ICCF; Peterson et al. 2004), discrete cross-correlation function (DCF; Edelson and Krolik 1988), and the z-transformed discrete cross-correlation function (zDCF; Alexander 1997).

The ICCF is perhaps the most straightforward way to compute a CCF between two unevenly sampled light curves. In this method, for each specified value of time lag, one of the two light curves is shifted by that time lag and its flux values are interpolated (often linearly) at the times of the second light curve. Often the ICCF is computed twice, once by fixing the first light curve and shifting and interpolating the second light curve and vice versa (White and Peterson 1994). The final ICCF is an average of the two CCFs and is thus not significantly biased towards the sampling of either of the two light curves. A DCF, on the other hand, only uses the actual data points without any interpolation (see, Edelson and Krolik 1988, for more details). A zDCF is based on the DCF with several modifications. It has been argued that zDCF can tease out weaker cross-correlation signals from data than the traditional DCF (see, Alexander 1997). All three methods have been used successfully within the context of AGN reverberation mapping but only rarely for TDE studies. 

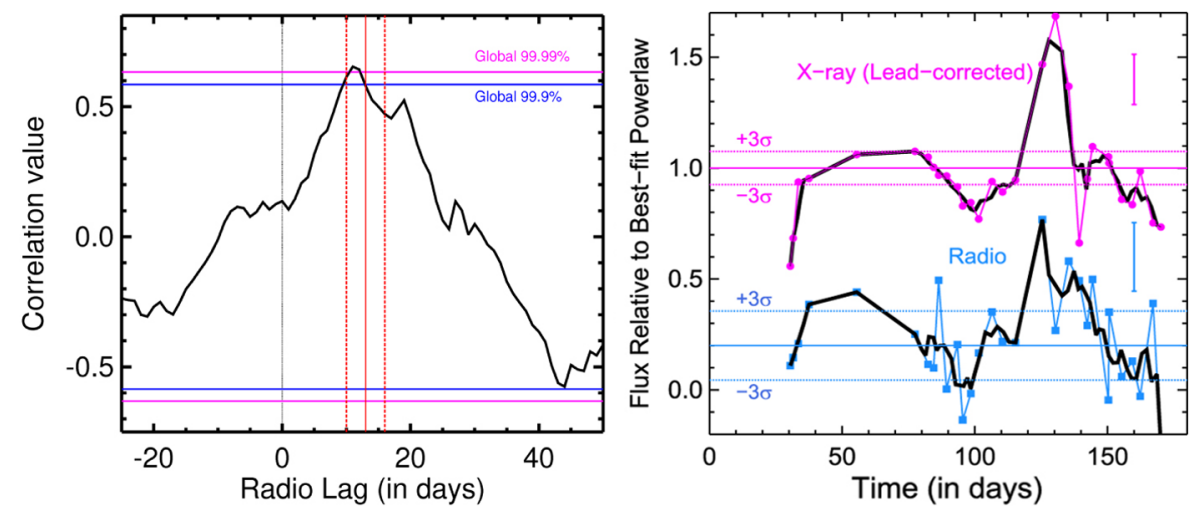

Fig. 6 Left: Cross-correlation function (CCF) between the soft X-ray (0.3-1 keV) and $16 \mathrm{GHz}$ radio data from ASASSN-14li. The horizontal blue and magenta lines are the global $99.9 \%$ and the $99.99 \%$ white noise statistical confidence contours for a blind search between -25 and 50 days (see Sect. 3.2 of Pasham and van Velzen 2018, for more details). The solid and the dashed vertical lines are the median and the $1 \sigma$ deviations $\left(13_{-3}^{+3}\right.$ days) derived by considering both the sampling and the measurement uncertainties in both the X-ray and radio light curves. Right: The relative X-ray (magenta) and radio (blue) light curves of ASASSN-14li obtained by dividing their corresponding best-fit power-law models. Both the light curves show the same variability pattern. The X-ray and radio fractional variability amplitudes on top of the power-law decay are $10 \% \pm 1 \%$ and $16 \% \pm 1 \%$, respectively. The mean flux levels are shown by the solid horizontal lines while the $\pm 3 \sigma$ variability contours derived using the methodology described in Romero-Cañizales et al. (2011) are indicated by dashed horizontal lines. The radio data have been vertically offset by -0.8 . The solid black curves are running averages over a 10 day window (except when the gap between observations in longer than 10 days). Typical $1 \sigma$ measurement uncertainties are shown as vertical bars. The X-ray data points shown here have been interpolated to match with the radio epochs (see Sect. 3.1 of Pasham and van Velzen 2018, for more analysis details) (adapted from Pasham and van Velzen 2018)

\subsection{Radio and X-Ray Cross Correlations}

Because of its panchromatic brightness, ASASSN-14li was monitored in the radio and the $\mathrm{X}$-ray (0.3-10 keV) wavebands roughly once every 3-10 days with a temporal baseline of a few hundred days (Pasham and van Velzen 2018). ASASSN-14li's X-ray spectrum was soft with very weak emission above $\sim 1 \mathrm{keV}$. These data sets provided the first opportunity to search for reverberation signals on timescale of tens of days from a TDE. Pasham and van Velzen (2018) extracted an ICCF between the X-ray and the $16 \mathrm{GHz}$ radio emission from ASASSN-14li and found that the emission at $16 \mathrm{GHz}$ lagged behind the soft/thermal $\mathrm{X}$-rays by roughly 13 days (see Fig. 6). Interestingly, both the fluctuations on top of the overall long-term decay and the long-term decay curves lagged by the same timescale. This suggested that the entire radio flux at $16 \mathrm{GHz}$ was responding to variations in the soft $\mathrm{X}$-ray (0.3-1.0 keV) brightness changes.

Based on this coupling between the radio and the soft X-rays and time-resolved radio spectral modeling, Pasham and van Velzen (2018) suggested that the radio emission from ASASSN-14li originates from a sub-relativistic jet that is regulated by the inner accretion disk which emits X-rays. Moreover, they showed using the correlation between the two wavebands and independently via modeling the time-resolved radio spectral energy distributions, that the accretion rate and the jet power are linearly coupled, i.e., the newly formed accretion disk is efficiently regulating the radio jet (for more details see Sect. 4.4 of Pasham and van Velzen 2018). Such a strong coupling between the soft X-rays, i.e., inner accretion flow, and the radio emission has never been seen before in an AGN or a supernova and thus 
may be a means to distinguish TDEs from TDE imposters. However in order to establish this as a common TDE phenomenon, similar studies of several events in the future are necessary. Nevertheless, this work demonstrated the use of TDE radio and X-ray observations to probe the earliest phases of jet formation which is currently only poorly constrained by observations.

\subsection{Optical/UV and X-Ray Cross Correlations}

In addition to radio-X-ray correlation studies, the inclusion of the optical and the UV wavebands in TDE photometric reverberation analysis can reveal insights into the physical origin of optical/UV light. Within the context of AGN, time lags between the X-ray and the optical/UV variations can arise from the following various physical scenarios (e.g., McHardy et al. 2016). Firstly, X-rays from near the black hole can scatter off material in the outer accretion disk or any other surrounding medium, lose energy, and get reprocessed into lowerenergy optical/UV photons (Edelson et al. 2015). In this case, fluctuations in the X-rays would lead the optical changes by a few days to a few tens of days depending on the distance (and thus the light travel time) to the outer disk/reprocessing region (Morgan et al. 2010). Alternatively, if the optical and the UV emission is dominated by the thermal emission from the accretion disk, two kinds of correlations/lags are possible. (1) If the X-rays are produced in a Corona by Compton up-scattering of the thermal near-UV "seed" photons (Reynolds and Nowak 2003), then the changes in the X-ray flux would lag behind the nearUV fluctuations by a fraction of a day to a few days depending on the size of the corona and the inner accretion disk (which are determined by the black hole mass, e.g., Arévalo et al. 2005, and accretion rate). (2) Also in this scenario, because the optical/UV originates directly from the disk, accretion rate perturbations can manifest as variations in optical and UV emission. As these perturbations propagate inwards in the disk on the local viscous timescale (Arévalo et al. 2008), they first trigger changes in the optical band followed by the UV and finally X-rays. Thus, we may expect the optical/UV emission to lead the X-rays by a few tens to millions of days depending on the black hole mass and the accretion rate (Shakura and Sunyaev 1973), see Figs. 8 and 9.

Due to its early identification as a compelling TDE, ASASSN-14li was monitored at a cadence of once every 3-7 days in the optical/UV bands for about 250 days. Motivated by optical/UV-X-ray photometric reverberation mapping of AGN (Edelson et al. 2015; McHardy et al. 2018), Pasham et al. (2017) performed cross-correlation analysis between the soft X-ray and the several of ASASSN-14li's available optical/UV light curves. They found evidence for the presence of similar flux variations in both the X-ray and optical/UV bands. In addition, the variability pattern in X-rays lagged behind those in the optical and the UV bands by roughly 32 days (see Fig. 7).

Based on the direction of the time lag (X-rays lagging the optical/UV), it appears that $\mathrm{X}$-ray reprocessing is not the dominant source of optical/UV emission in ASASSN-14li. Furthermore, the seed photon scenario described above is also unlikely because the expected UV lead times for a $10^{6.5 \pm 0.6} \mathrm{M}_{\odot}$ black hole-implied from host galaxy properties-are only a few thousand to a few tens of thousand seconds, which is an order of magnitude shorter than the observed lead time. Also, in ASASSN-14li, there is no evidence for a Corona (nonthermal emission) in the high quality X-ray spectra obtained during the first $\sim 300 \mathrm{~d}$ of monitoring.

The observed lags are also unlikely to be due to viscous time delays in a "standard" circular Shakura-Sunyaev accretion disk (Shakura and Sunyaev 1973). ASASSN-14li's peak bolometric luminosity of $\sim 10^{44} \mathrm{erg} \mathrm{s}^{-1}$ (Holoien et al. 2016) implies a peak Eddington ra- 

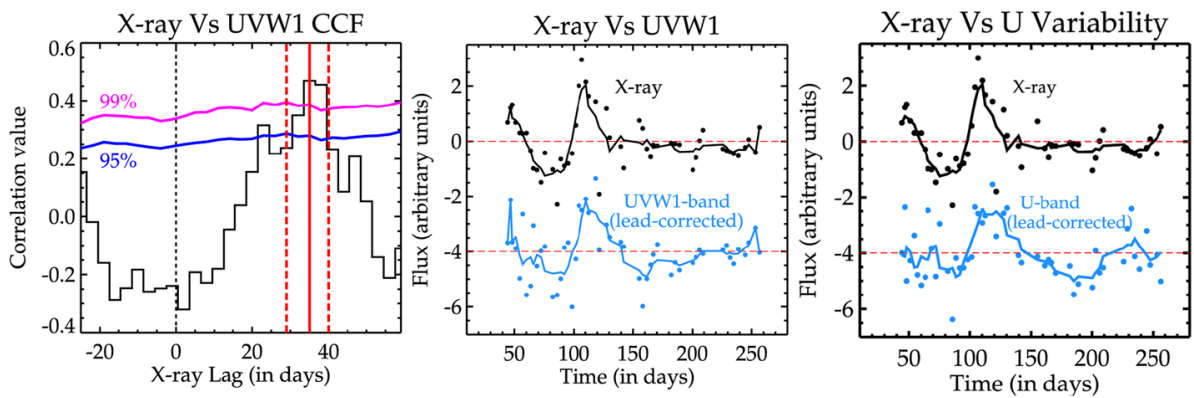

Fig. 7 Left: Cross-correlation function (CCF) between the soft X-ray (0.3-1 keV) and the UV (UVW1 filter of Neil Gehrels Swift observatory) light curves from ASASSN-14li. The blue and magenta lines are the statistical significance contours (see Pasham et al. 2017, for more details). The vertical red lines have the same meaning as in the left panel of Fig. 6. Middle and Right: UV and the optical (U band) variability features are compared to the X-ray variations. The UVW1/U-band light curves are offset by 35 days and the X-ray light curve is interpolated at the lead-corrected UVW1/U epochs. In both panels the observed X-ray and UVW1/U light curves were de-trended by subtracting a smooth function, leaving only the variability features. The solid curves are a running average of five neighboring points, to guide the eye (adapted from Pasham et al. 2017)
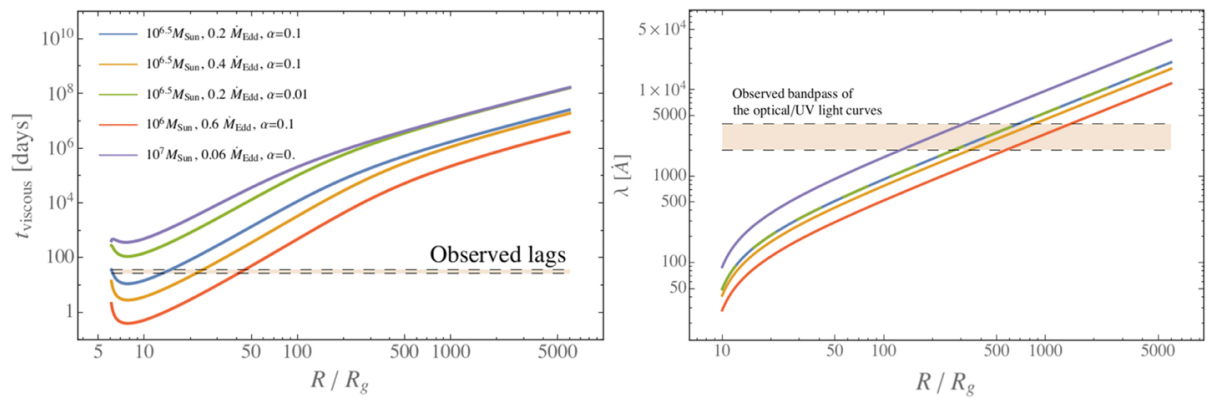

Fig. 8 The dependence of the local viscous timescale (left) and the wavelength of peak emission (right) on the radial distance from the black hole in a Shakura-Sunyaev accretion disk for a set of black hole masses, Eddington ratios and $\alpha$-viscosity parameter values. The observed timescale of $\sim 30$ days is inconsistent with a circular disk and argues for an accretion flow in ASASSN-14li that is distinct from AGN accretion disks (adapted from Pasham et al. 2017)

tio of $0.2_{-0.2}^{+0.7} \dot{M}_{\text {Edd }}$ (where $\dot{M}_{\text {Edd }}$ is the Eddington rate) for a black hole in the mass range of $10^{6.5 \pm 0.6} M_{\odot}$ (e.g., Holoien et al. 2016; Pasham et al. 2019). At such sub-Eddington rates, the newly formed accretion disk can be described by a geometrically thin, optically thick disk model of Shakura and Sunyaev (1973). For the observed lag timescale of $\sim 30$ days to correspond to viscous delay in a disk the optical/UV emission should originate from within a radius of $50 R_{g}$ (see the left panel of Fig. 8). However, the bulk of the optical/UV emission in the observed Swift bandpass (1500-6000 ̊) originates from beyond a few hundred gravitational radii (see the right panel of Fig. 8) and at these larger radii the viscous timescale and thus the time lags are orders of magnitude longer. This discrepancy between the emission radius, emission bandpass and corresponding viscous timescales rules out the standard, thin, circular disk solution.

This discrepancy can be resolved in a few of ways. First, the disrupting black hole could be a few orders of magnitude smaller than $10^{6} M_{\odot}$, i.e., a so-called intermediate-mass black hole. This would make the accretion flow super-Eddington and produce slim disks 
Fig. 9 The colored curves (green, blue and red) show the dependence of viscous timescale on wavelength of emission for a standard, circular thin disk. The observed time lags, shown as blue data points, are orders of magnitude faster than expected from a circular thin disk. The viscous time lags in a simplistic elliptical accretion disk described in Guillochon et al. (2014) (black curve) are shorter and are broadly consistent with the observed optical/UV-X-ray time lags in ASASSN-14li (adapted from Pasham et al. 2017)

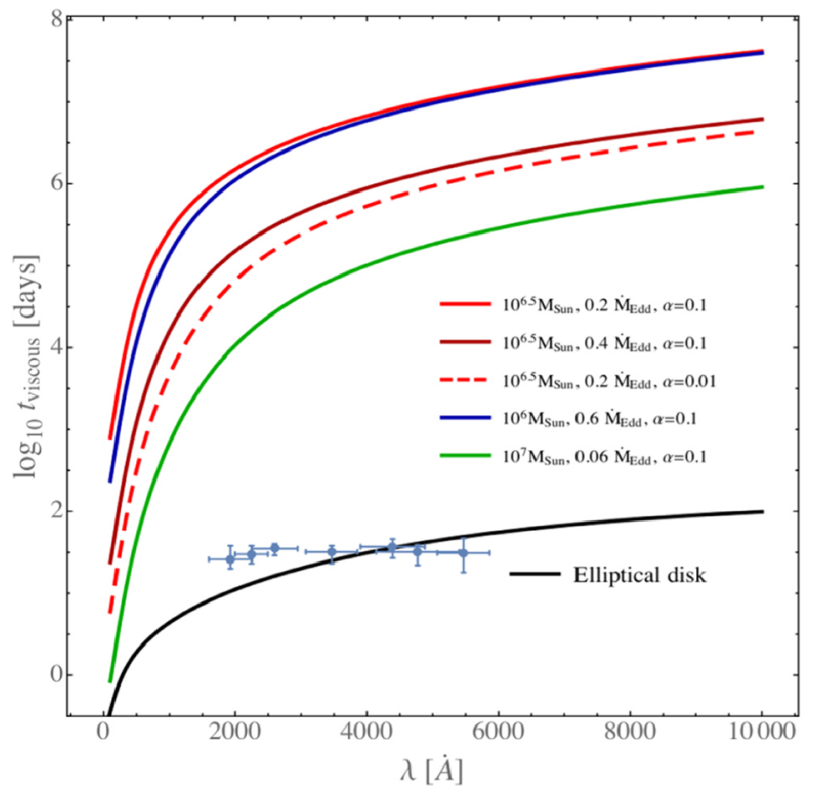

(Abramowicz et al. 1988) which can yield faster viscous timescales, i.e., comparable to the optical/UV-X-rays lags observed in ASASSN-14li. Alternately, an elliptical accretion disk would also shorten the viscous timescales (see Fig. 9).

In any case, it is clear that at least in the quintessential TDE ASASSN-14li the optical/UV emission vary with respect to the central X-rays in a unique manner. It remains to be seen if this behavior is typical in TDEs. If so, these distinguishing "echo" signals could be a means to separate genuine TDEs from imposters, viz., AGN flares and unusual supernovae. We note that cross-correlation studies like the ones carried out for ASASSN-14li require highcadence monitoring over a long temporal baseline (once every few days for several months) in multiple wavebands, and therefore may not be feasible in many cases.

\section{X-Ray Quasi-periodic Oscillations from TDEs}

Quasi-periodic oscillations (QPOs) in the X-ray flux with centroid frequencies between a few $\mathrm{mHz}$ to several hundreds of $\mathrm{Hz}$ have now been observed from several stellar-mass black hole binaries (McClintock and Remillard 2006). These are systems where the central compact object has a mass between 5 and $20 \mathrm{M}_{\odot}$ and draws material from a non-degenerate companion star. X-ray QPOs in stellar-mass black hole binaries often appear when the black hole goes into an outburst during which the overall X-ray flux can increase by orders of magnitude for a brief duration of a few weeks to months (e.g., Homan et al. 2005; Remillard et al. 2002). While the exact origin of these X-ray modulations is unclear, they are generally thought to be associated with the dynamics of hot gas in the innermost regions of the accretion flow (see McClintock and Remillard 2006 for a review). In some systems, the spins of the central black holes have been estimated under the assumption that the QPOs are associated with test particle orbits predicted from general relativity (Franchini et al. 2017; Motta et al. 2014). 
Table 3 Summary of the currently known TDE quasi-periodic oscillations (QPOs)

\begin{tabular}{|c|c|c|c|}
\hline Flare name & $\begin{array}{l}\text { Centroid } \\
\text { frequency }\end{array}$ & $\begin{array}{l}\text { Fractional } \\
\text { rms* }\end{array}$ & Notes \\
\hline SwJ1644+57 & $4.8 \pm 0.3 \mathrm{mHz}$ & $3-4 \%$ & $\begin{array}{l}\text { QPO was present only within the } \\
\text { first two weeks after the flare } \\
\text { was discovered }\end{array}$ \\
\hline 2XMM J123103.2+110648 & $\sim 7.3 \times 10^{-5} \mathrm{~Hz}$ & $20-60 \%$ & $\begin{array}{l}\text { The optical spectrum is unusual } \\
\text { compared to typical TDEs. } \\
\text { Could be an AGN flare }\end{array}$ \\
\hline ASASSN-14li & $7.65 \pm 0.4 \mathrm{mHz}$ & $3-40 \%$ & $\begin{array}{l}\text { Stable QPO lasting for at least } \\
500 \text { d after the flare was } \\
\text { discovered }\end{array}$ \\
\hline
\end{tabular}

\footnotetext{
${ }^{\dagger}$ The centroid frequency of the X-ray QPO

*The fraction root-mean-squared amplitude of the QPO in percentage of mean flux
}

A wealth of X-ray timing studies now suggest that supermassive black holes (SMBHs) are scaled-up versions of stellar-mass black holes in some aspects (e.g., McHardy et al. 2006; Markowitz et al. 2003). For example, it has been shown that the X-ray power density spectra of both SMBHs in AGN and stellar-mass black holes are qualitatively similar and that certain timescales within the power spectra scale inversely with the black hole mass after correcting for the accretion rate (McHardy et al. 2006; Markowitz et al. 2003; but also see Done and Gierliński 2005). Under this black hole unification paradigm, even SMBHs should exhibit QPOs but with frequencies that are $10^{5-7}$ times lower depending on the exact SMBH mass. However, in spite of several searches, robust evidence for X-ray QPOs from SMBHs in AGN has been scarce (see, however, Gierliński et al. 2008; Alston et al. 2014). This could be because of a dearth of highly sensitive observations or that QPOs are intrinsically absent in steadily accreting SMBHs (see Vaughan and Uttley 2005 for details).

On the other hand, TDEs are different from steadily accreting SMBHs and to some degree are similar to stellar-mass black hole binaries when they go into outburst. This is because in both stellar-mass black hole outbursts and TDEs starving black holes are suddenly fed at a high rate. Thus, the natural expectation is that some of the same phenomena, viz., X-ray QPOs, that occur in stellar-mass black hole outbursts should also appear in TDEs. There have been three cases where X-ray QPOs have been reported from TDE (candidates). These include the relativistic TDE SwJ1644+57 (Reis et al. 2012), the super soft X-ray flare 2XMM J123103.2+110648 (Lin et al. 2015), and the thermal TDE ASASSN-14li (Pasham et al. 2019). The observed properties of these three QPOs are summarized in Table 3.

The case of ASASSN-14li is particularly interesting. Because of its widespread interest ASASSN-14li was monitored for several years at multiple wavelengths. In particular, the flare was detectable with Swift's X-Ray Telescope (XRT) for multiple years after its discovery and several deeper exposures were obtained with Chandra and XMM-Newton during this time. An average power density spectrum of the Chandra and the XMM-Newton data segments spread over the first $500 \mathrm{~d}$ reveals a prominent feature at a frequency of $7.65 \pm 0.4$ $\mathrm{mHz}$ (Fig. 10, left panel). The feature was surprisingly stable for over 300,000 cycles and had a fractional root-mean-squared amplitude of $40 \%$ around day 450 . The stability for such large number of cycles while the flux changed by an order of magnitude suggests an origin that is tied to the fundamental properties, viz., the mass and the spin of the disrupting black hole (Pasham et al. 2019). Using a black hole mass implied from host galaxy properties, i.e., 

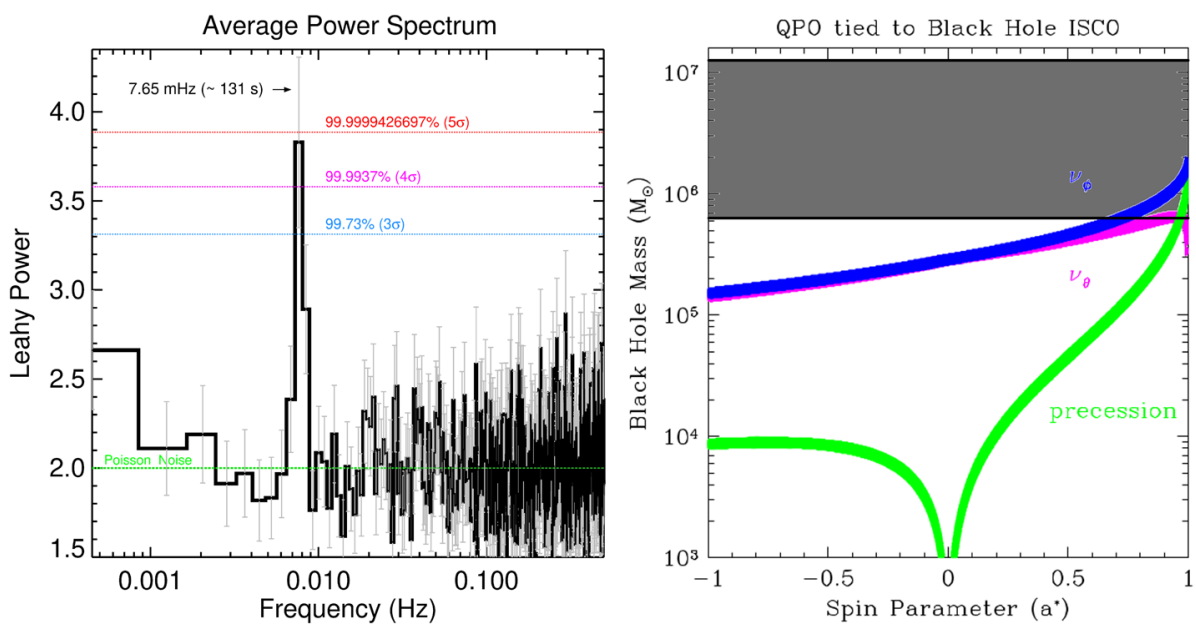

Fig. 10 Left: Average X-ray (0.3-1 keV) power density spectrum of ASASSN-14li using data segments spread over $500 \mathrm{~d}$ (adapted from Pasham et al. 2019). The global statistical confidence levels and the Poisson noise level are shown as dashed horizontal lines. The strongest features in the spectrum is at $7.65 \pm 0.4 \mathrm{mHz}$. Right: Black hole mass vs dimensionless spin parameter contours estimates by assuming the QPO is associated with test particle orbits at the innermost stable circular orbit (ISCO). Contours corresponding to three particle frequencies: Keplerian frequency $\left(v_{\phi}\right.$, blue), vertical epicyclic frequency $\left(v_{\theta}\right.$, magenta) and LenseThirring precession $\left(v_{\phi}-v_{\theta}\right.$, green) at the ISCO are shown. At the ISCO the radial epicyclic frequency $\left(v_{r}\right)$ is zero and the periastron precession frequency $\left(v_{\phi}-v_{r}\right)$ is thus equal to the Keplerian frequency. The widths of these contours reflect the QPO's width of $0.7 \mathrm{mHz}$ (upper limit). The shaded grey rectangular area represents ASASSN-14li's black hole mass range $\left(10^{5.8-7.1} M_{\odot}\right)$ estimated from its host galaxy scaling relations. Within this mass range, the only formal solutions are the ones that require the spin parameter to be greater than 0.7 . Choosing a radius larger than the ISCO only pushes this lower limit to a higher value

based on the scaling laws between the black hole mass and the host's stellar velocity dispersion and the luminosity, and associating the QPO period to the various particle frequencies predicted from general relativity, Pasham et al. (2019) constrained the dimensionless spin parameter of the disrupting black hole to be at least 0.7 (Fig. 10, right panel). This study presented a new means to constrain spins of several SMBHs in the future when they tidally disrupt stars. On the other hand, the contours in the right panel of Fig. 10 can also be used to set an absolute upper limit on black hole mass. For ASASSN-14li this upper limit corresponds to $2 \times 10^{6} M_{\odot}$. For future events with QPOs this could be a straightforward technique to identify the long-sought intermediate-mass black holes (masses in $10^{2-5} M_{\odot}$ ) whose QPO timescales would be less than a few tens of seconds.

Historically, not many X-ray bright TDEs have been followed-up with highly sensitive Xray observations as a majority of them were discovered in archival searches (e.g., Komossa and Bade 1999; Esquej et al. 2007 and see Komossa 2015 and references therein). But, several of the events that did have detailed X-ray observations showed QPOs. While the number of such X-ray TDEs with QPOs is currently small, it remains plausible that QPOs may have been missed in several earlier TDEs. If ASASSN-14li is indeed a "poster child" for TDEs and the observed X-ray QPOs are typical in these systems, then this opens a new opportunity to use TDEs to build a census of black hole mass. An X-ray observatory with an effective area of the order of a square meter will be transformational for this purpose. For instance, simulations using spectral responses of the Wide Field Image (WFI) and the X-ray Integral Field Unit (X-IFU) on board the anticipated meter-class X-ray observatory Athena suggest that ASASSN-14li like QPOs could be detected out to a redshift of 2 (Fig. 11). 

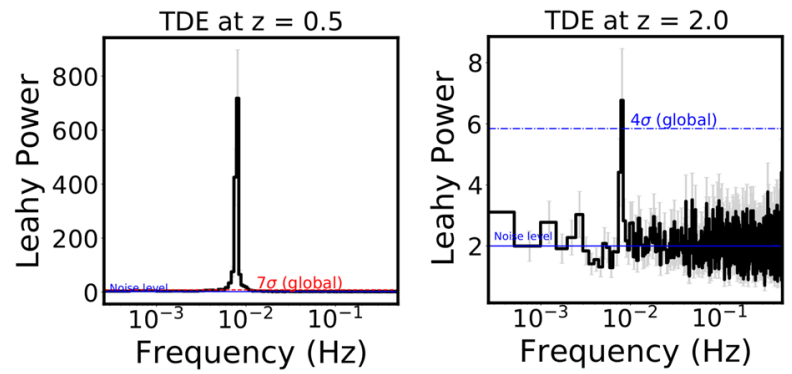

Fig. 11 Simulated power density spectra of an ASASSN-14li-like TDE at various redshifts using Athena's Wide Field Imager (WFI). For each of these simulations we used an exposure time of $32 \mathrm{ks}$ and a QPO fractional rms amplitude of $40 \%$ (similar to ASASSN-14li) whose value remains constant with energy within 0.3-2 keV bandpass. Statistical confidence contours and the Poisson noise level of 2 are shown as dashed horizontal lines. The time dilation effect due to the redshift and the potential well of the source on the centroid frequency of the QPO was neglected as it will not alter the results significantly. Assuming ASASSN-14li-like behavior, TDE X-ray QPOs could be detected out to a redshift of roughly 2 with a meter-class X-ray telescope

The prospect of detecting QPOs from TDEs at cosmological distances is very exciting because it would allow us to use these signals to constrain spins of a large number of SMBHs and also construct SMBH spin distributions at various redshifts. On the other hand, the QPOs themselves are excellent probes of accretion at these redshifts. Spin distributions are especially important to constrain the models for growth of SMBHs. For example, it has been predicted that if SMBHs grew primarily via mergers with other black holes, their spin distribution should be skewed towards low spin (e.g., Berti and Volonteri 2008). This is because mergers tend to deposit angular momentum along random orientations which tends to generally spin down the remnant hole. On the other hand, growth by steady accretion tends to add angular momentum along the spin axis of the hole and thus tends to spin up the SMBH. Thus TDE QPOs and the resulting spin constraints could provide a novel opportunity to understand the dominant growth channel of SMBHs.

\section{X-Ray Spectroscopy: Relativistic Reverberation}

$\mathrm{X}$-ray light echoes probe the smallest scales around the black hole. X-ray reverberation is commonly seen in AGN systems, most of which are sub-Eddington systems consisting of an isotropically-emitting X-ray corona that irradiates the thin accretion disc (e.g. De Marco et al. 2013; Uttley et al. 2014). This produces fluorescence lines with a time delay corresponding to a light travel distance of a few gravitational radii (e.g. Zoghbi et al. 2012; Kara et al. 2016a). The reverberation lags found in AGN can probe the extent of the corona and the inner radius of the thin accretion disc (e.g. Wilkins et al. 2016; Taylor and Reynolds 2018).

Thus far, only one TDE is bright and variable enough (on timescales of hundreds to thousands of seconds) to enable the detection of X-ray time lags. This source is Swift J1644+57, the most well-observed hard X-ray TDE. In this source, Kara et al. (2016b) discovered an emission line at $8 \mathrm{keV}$, which was seen to lag behind the continuum by 100 seconds. If associated with the $\mathrm{Fe} \mathrm{K} \alpha$ line (the line with the highest fluorescent yield in the X-ray spectrum), then the line must be highly blueshifted.

The extremely high isotropic luminosity and the detection of strong radio emission from Swift J1644+57 suggest that this is a TDE with a jet along our line of sight, and a face-on accretion flow. The highly blueshifted iron $\mathrm{K}$ line and corresponding reverberation lag can be 
well described by reprocessing off of a Compton thick, outflowing disc wind with a velocity of $>0.1 c$ and $\sim 30 \mathrm{deg}$ opening angle (Kara et al. 2016b; Lu et al. 2017; Thomsen et al. 2019 ). The iron $\mathrm{K}$ lag is marginally better described ( $>98 \%$ confidence) by an asymmetrically broadened line with some gravitational redshifted over a symmetric Gaussian. This suggested that the reprocessing occurred close to the black hole, and therefore at least some of the X-ray emitting continuum is isotropically emitting (Kara et al. 2016b). This was also supported by the short $100 \mathrm{~s}$ lag, though, Lu et al. (2017) point out that if the iron line band also contains emission from the primary continuum source (an effect known as 'dilution'), then the iron $\mathrm{K}$ reprocessing region could be further from the black hole, and thus do not rule out that the X-rays are produced in the slow sheath of a relativistic jet.

To conclude, we have shown that X-ray reverberation lags probe the accretion flow structure around black holes, and can be used to probe the velocity and geometrical structure of ultrafast outflows in TDEs.

\section{Concluding Remarks and Outlook}

Since the strength of a reverberation signal depends on the availability of material to reflect or reprocess the transient emission, we anticipate these signal are not universal in TDEs. Indeed, dust echoes are not detected for every TDE (e.g. van Velzen et al. 2016; Jiang et al. 2021) and the extreme coronal lines seen in SDSS J0952+2143 (Fig. 3) and several other SDSS sources have never been observed in follow-up optical spectra of TDEs detected in other imaging surveys. Furthermore, since not every TDE is X-ray bright (e.g. Auchettl et al. 2017), the X-ray cross-correlation and timing signatures discussed this review also remain relatively rare, with detections in just one (either ASASSN-14li or Swift J1644) or a handful of sources: see Table 3 .

While they are not common to the full TDE population, the detection of reverberation signals is important since they provide a novel way to study the TDE emission mechanism and these signals can be used to select TDEs that could be missed by traditional photometric searches for TDEs. The events selected based on the detection of galaxies with extreme coronal lines are well-suited to recover a subset of TDEs that occur in gas rich circumnuclear environments, while a search for IR echoes can reveal TDEs in galaxies with a large amount of dust extinction to the center (e.g., Wang et al. 2018; Mattila et al. 2018). We should note that IR follow-up observations of TDE candidates in AGN have yielded dust echoes that are an order of magnitude more luminous compared to IR echoes in TDEs from quiescent galaxies (Table 1), implying that a blind search for IR echoes could be dominated by flares in AGN (see also the discussion at the end of 2.3).

Both the IR echoes and the detection of coronal lines can be used to probe the energy produced at EUV wavelengths (which cannot be observed directly due to absorption by neutral hydrogen). In most cases, the observed optical/UV or X-ray luminosity is lower than the energy inferred from the reverberation signals. This provides support for models that explain the optical/UV emission of TDEs with an accretion disk (Cannizzo et al. 1990; Dai et al. 2018; van Velzen et al. 2019; Mummery and Balbus 2020), since for black holes in the mass range $M_{\mathrm{BH}} \sim 10^{6-7} M_{\odot}$ these disk are expected to emit most of their energy in the EUV regime, see also the Emission Mechanisms Chapter (Roth et al. 2020) of this journal.

While the IR echoes are agnostic to the source that is heating the dust, the crosscorrelation signals detected for ASASSN-14li (Sect. 4) appear to be unique to TDEs and can thus be used to help discriminate TDEs from impostors such as AGN flares, see the Imposters Chapter (Zabludoff et al. 2021) of this journal for further discussion. In particular 
the detection of a QPO in the X-ray light curve of ASASSN-14li is truly spectacular, providing new ways to constrain the spin of the black hole that disrupted the star. The relativistic reverberation signal (Sect. 6) detected for the jetted TDE Swift J1644+57 is another unique signature which probes the geometry of the inter accretion flow and the base of the jet.

Clearly the detection of more multi-wavelength cross-correlations and QPOs is very much anticipated. The current detection rate of such events is $\sim 1$ per decade. While recently the detection rate of optical TDEs has increased significantly, to dozens of new sources per year (e.g., van Velzen et al. 2021), few of these have well-sampled X-ray light curves similar to ASASSN-14li (one notable exception is AT2018fyk, see Wevers et al. 2019, 2021). This lack of useful X-ray light curves is mainly explained by the low X-ray flux of opticallyselected TDEs, but is also due to a scarcity of X-ray follow-up resources. An important improvement would be a new X-ray mission that is dedicated to high-cadence observations, such as Einstein Probe (Yuan et al. 2015). As discussed in Sect. 5, X-ray observations with a meter-class X-ray observatory such as Athena would be able to detect QPOs from TDEs to $z \sim 2$, thus constraining the spin of black holes in the cosmic epoch when they are expected to be rapidly growing.

At the time of writing, the WISE satellite continues to monitor the near-IR sky (3-4 $\mu \mathrm{m})$ with a cadence of 6 months, thus providing an excellent dataset that can be used to obtain detections (or upper limits) of dust echoes for every transient detected in the last decade. The (potential) detection of dust reprocessing using ground-based $i$-band observations (Stein et al. 2020) and $K$-band observation (Mattila et al. 2018; Kool et al. 2020) is also an encouraging result, because this allows for a much higher cadence than the scanning pattern of WISE. In the near future, observations with JWST could yield a detailed spectroscopic view of dust heated by TDEs.

Open Access This article is licensed under a Creative Commons Attribution 4.0 International License, which permits use, sharing, adaptation, distribution and reproduction in any medium or format, as long as you give appropriate credit to the original author(s) and the source, provide a link to the Creative Commons licence, and indicate if changes were made. The images or other third party material in this article are included in the article's Creative Commons licence, unless indicated otherwise in a credit line to the material. If material is not included in the article's Creative Commons licence and your intended use is not permitted by statutory regulation or exceeds the permitted use, you will need to obtain permission directly from the copyright holder. To view a copy of this licence, visit http://creativecommons.org/licenses/by/4.0/.

\section{References}

M.A. Abramowicz, B. Czerny, J.P. Lasota, E. Szuszkiewicz, Slim accretion disks. Astrophys. J. 332 , 646 (1988). https://doi.org/10.1086/166683

T. Alexander, Is AGN variability correlated with other AGN properties? in ZDCF Analysis of Small Samples of Sparse Light Curves, vol. 218, ed. by D. Maoz, A. Sternberg, E.M. Leibowitz (1997), p. 163. https:// doi.org/10.1007/978-94-015-8941-3_14

K.D. Alexander, S. van Velzen, A. Horesh, B.A. Zauderer, Radio properties of tidal disruption events. Space Sci. Rev. 216(5), 81 (2020). https://doi.org/10.1007/s11214-020-00702-w

W.N. Alston, J. Markevičiūte, E. Kara, A.C. Fabian, M. Middleton, Detection of a QPO in five XMM-Newton observations of RE J1034+396. Mon. Not. R. Astron. Soc. 445, 16-20 (2014). https://doi.org/10.1093/ mnrasl/slu 127

I. Arcavi, A. Gal-Yam, M. Sullivan, Y.-C. Pan, S.B. Cenko, A. Horesh, E.O. Ofek, A. De Cia, L. Yan, C.-W. Yang, D.A. Howell, D. Tal, S.R. Kulkarni, S.P. Tendulkar, S. Tang, D. Xu, A. Sternberg, J.G. Cohen, J.S. Bloom, P.E. Nugent, M.M. Kasliwal, D.A. Perley, R.M. Quimby, A.A. Miller, C.A. Theissen, R.R. Laher, A continuum of $\mathrm{H}$ - to He-rich tidal disruption candidates with a preference for E+A galaxies. Astrophys. J. 793, 38 (2014). https://doi.org/10.1088/0004-637X/793/1/38

P. Arévalo, I. Papadakis, B. Kuhlbrodt, W. Brinkmann, X-ray to UV variability correlation in MCG-6-30-15. Astron. Astrophys. 430, 435-442 (2005). https://doi.org/10.1051/0004-6361:20041801 
P. Arévalo, P. Uttley, S. Kaspi, E. Breedt, P. Lira, I.M. McHardy, Correlated X-ray/optical variability in the quasar MR2251-178. Mon. Not. R. Astron. Soc. 389, 1479-1488 (2008). https://doi.org/10.1111/j.13652966.2008.13719.x

K. Auchettl, J. Guillochon, E. Ramirez-Ruiz, New physical insights about tidal disruption events from a comprehensive observational inventory at X-ray wavelengths. Astrophys. J. 838, 149 (2017). https:// doi.org/10.3847/1538-4357/aa633b

R. Barvainis, Hot dust and the near-infrared bump in the continuum spectra of quasars and active galactic nuclei. Astrophys. J. 320, 537-544 (1987). https://doi.org/10.1086/165571

E. Berti, M. Volonteri, Cosmological black hole spin evolution by mergers and accretion. Astrophys. J. 684, 822-828 (2008). https://doi.org/10.1086/590379

P.K. Blanchard, M. Nicholl, E. Berger, J. Guillochon, R. Margutti, R. Chornock, K.D. Alexander, J. Leja, M.R. Drout, PS16dtm: A tidal disruption event in a narrow-line Seyfert 1 galaxy. Astrophys. J. 843, 106 (2017). https://doi.org/10.3847/1538-4357/aa77f7

J.S. Bloom, D. Giannios, B.D. Metzger, S.B. Cenko, D.A. Perley, N.R. Butler, N.R. Tanvir, A.J. Levan, P.T. O’Brien, L.E. Strubbe, F. De Colle, E. Ramirez-Ruiz, W.H. Lee, S. Nayakshin, E. Quataert, A.R. King, A. Cucchiara, J. Guillochon, G.C. Bower, A.S. Fruchter, A.N. Morgan, A.J. van der Horst, A possible relativistic jetted outburst from a massive black hole fed by a tidally disrupted star. Science 333, 203 (2011). https://doi.org/10.1126/science.1207150

E.M. Cackett, C.-Y. Chiang, I. McHardy, R. Edelson, M.R. Goad, K. Horne, K.T. Korista, Accretion disk reverberation with Hubble space telescope observations of NGC 4593: Evidence for diffuse continuum lags. Astrophys. J. 857, 53 (2018). https://doi.org/10.3847/1538-4357/aab4f7

J.K. Cannizzo, H.M. Lee, J. Goodman, The disk accretion of a tidally disrupted star onto a massive black hole. Astrophys. J. 351, 38-46 (1990). https://doi.org/10.1086/168442

R. Chatterjee, A.P. Marscher, S.G. Jorstad, A.R. Olmstead, I.M. McHardy, M.F. Aller, H.D. Aller, A. Lähteenmäki, M. Tornikoski, T. Hovatta, Disk-jet connection in the radio galaxy 3C 120. Astrophys. J. 704(2), 1689-1703 (2009). https://doi.org/10.1088/0004-637X/704/2/1689

L. Dai, J.C. McKinney, N. Roth, E. Ramirez-Ruiz, M.C. Miller, A unified model for tidal disruption events. Astrophys. J. 859, 20 (2018). https://doi.org/10.3847/2041-8213/aab429

B.B. Dai, X.W. Shu, N. Jiang, L.M. Dou, D.Z. Liu, C.W. Yang, F.B. Zhang, T.G. Wang, Compact radio emission from nearby galaxies with mid-infrared nuclear outbursts. Astrophys. J. Lett. 896(2), 27 (2020). https://doi.org/10.3847/2041-8213/ab97ac

B. De Marco, G. Ponti, G. Miniutti, T. Belloni, M. Cappi, M. Dadina, T. Muñoz-Darias, Time lags in the ultraluminous X-ray source NGC 5408 X-1: Implications for the black hole mass. Mon. Not. R. Astron. Soc. 436, 3782-3791 (2013). https://doi.org/10.1093/mnras/stt1853

K.P. Dere, E. Landi, P.R. Young, G. Del Zanna, M. Landini, H.E. Mason, CHIANTI - An atomic database for emission lines. IX. Ionization rates, recombination rates, ionization equilibria for the elements hydrogen through zinc and updated atomic data. Astron. Astrophys. 498(3), 915-929 (2009). https://doi.org/10. 1051/0004-6361/200911712

C. Done, M. Gierliński, Scaling variability from stellar to supermassive black holes. Mon. Not. R. Astron. Soc. 364, 208-216 (2005). https://doi.org/10.1111/j.1365-2966.2005.09555.x

M.A. Dopita, R.S. Sutherland, Astrophysics of the Diffuse Universe (2003)

L. Dou, T-g. Wang, N. Jiang, C. Yang, J. Lyu, H. Zhou, Long fading mid-infrared emission in transient coronal line emitters: Dust echo of a tidal disruption flare. Astrophys. J. 832, 188 (2016). https://doi. org/10.3847/0004-637X/832/2/188

L. Dou, T. Wang, L. Yan, N. Jiang, C. Yang, R.M. Cutri, A. Mainzer, B. Peng, Discovery of a mid-infrared echo from the TDE candidate in the nucleus of ULIRG F01004-2237. Astrophys. J. Lett. 841(1), 8 (2017). https://doi.org/10.3847/2041-8213/aa7130

B.T. Draine, H.M. Lee, Optical properties of interstellar graphite and silicate grains. Astrophys. J. 285, 89-108 (1984). https://doi.org/10.1086/162480

E. Dwek, The infrared echo of a type II supernova with a circumstellar dust shell: Applications to SN 1979c and SN 1980k. Astrophys. J. 274, 175-183 (1983). https://doi.org/10.1086/161435

R.A. Edelson, J.H. Krolik, The discrete correlation function: A new method for analyzing unevenly sampled variability data. Astrophys. J. 333, 646 (1988). https://doi.org/10.1086/166773

R. Edelson, J.M. Gelbord, K. Horne, I.M. McHardy, B.M. Peterson, P. Arévalo, A.A. Breeveld, G. De Rosa, P.A. Evans, M.R. Goad, G.A. Kriss, W.N. Brandt, N. Gehrels, D. Grupe, J.A. Kennea, C.S. Kochanek, J.A. Nousek, I. Papadakis, M. Siegel, D. Starkey, P. Uttley, S. Vaughan, S. Young, A.J. Barth, M.C. Bentz, B.J. Brewer, D.M. Crenshaw, E. Dalla Bontà, A. De Lorenzo-Cáceres, K.D. Denney, M. Dietrich, J. Ely, M.M. Fausnaugh, C.J. Grier, P.B. Hall, J. Kaastra, B.C. Kelly, K.T. Korista, P. Lira, S. Mathur, H. Netzer, A. Pancoast, L. Pei, R.W. Pogge, J.S. Schimoia, T. Treu, M. Vestergaard, C. Villforth, H. Yan, Y. Zu, Space telescope and optical reverberation mapping project. II. Swift and HST reverberation mapping of the accretion disk of NGC 5548. Astrophys. J. 806, 129 (2015). https://doi.org/10.1088/ 0004-637X/806/1/129 
P. Esquej, R.D. Saxton, M.J. Freyberg, A.M. Read, B. Altieri, M. Sanchez-Portal, G. Hasinger, Candidate tidal disruption events from the XMM-Newton slew survey. Astron. Astrophys. 462, 49-52 (2007). https:// doi.org/10.1051/0004-6361:20066072

A. Franchini, S.E. Motta, G. Lodato, Constraining black hole spins with low-frequency quasi-periodic oscillations in soft states. Mon. Not. R. Astron. Soc. 467, 145-154 (2017). https://doi.org/10.1093/mnras/ stw3363

P. Gandhi, K. Makishima, M. Durant, A.C. Fabian, V.S. Dhillon, T.R. Marsh, J.M. Miller, T. Shahbaz, H.C. Spruit, Rapid optical and X-ray timing observations of GX 339-4: Flux correlations at the onset of a low/hard state. Mon. Not. R. Astron. Soc. 390, 29-33 (2008). https://doi.org/10.1111/j.1745-3933. 2008.00529.x

R. Genzel, C.J. Cesarsky, Extragalactic results from the infrared space observatory. Annu. Rev. Astron. Astrophys. 38, 761-814 (2000). https://doi.org/10.1146/annurev.astro.38.1.761

M. Gierliński, M. Middleton, M. Ward, C. Done, A periodicity of $\sim 1$ hour in X-ray emission from the active galaxy RE J1034+396. Nature 455, 369-371 (2008). https://doi.org/10.1038/nature07277

B.A. Groves, M.A. Dopita, R.S. Sutherland Dusty, Radiation pressure-dominated photoionization. I. Model description, structure, and grids. Astrophys. J. Suppl. Ser. 153(1), 9-73 (2004). https://doi.org/10.1086/ 421113

J. Guillochon, H. Manukian, E. Ramirez-Ruiz, PS1-10jh: The disruption of a main-sequence star of near-solar composition. Astrophys. J. 783(1), 23 (2014). https://doi.org/10.1088/0004-637X/783/1/23

K. Heng, R. McCray, Balmer-dominated shocks revisited. Astrophys. J. 654(2), 923-937 (2007). https://doi. org/10.1086/509601

T.W.-S. Holoien, C.S. Kochanek, J.L. Prieto, K.Z. Stanek, S. Dong, B.J. Shappee, D. Grupe, J.S. Brown, U. Basu, J.F. Beacom, D. Bersier, J. Brimacombe, A.B. Danilet, E. Falco, Z. Guo, J. Jose, G.J. Herczeg, F. Long, G. Pojmanski, G.V. Simonian, D.M. Szczygieł, T.A. Thompson, J.R. Thorstensen, R.M. Wagner, P.R. Woźniak, Six months of multiwavelength follow-up of the tidal disruption candidate ASASSN-14li and implied TDE rates from ASAS-SN. Mon. Not. R. Astron. Soc. 455, 2918-2935 (2016). https://doi. org/10.1093/mnras/stv2486

J. Homan, J.M. Miller, R. Wijnands, M. van der Klis, T. Belloni, D. Steeghs, W.H.G. Lewin, High- and lowfrequency quasi-periodic oscillations in the X-ray light curves of the black hole transient H1743-322. Astrophys. J. 623, 383-391 (2005). https://doi.org/10.1086/424994

N. Jiang, L. Dou, T. Wang, C. Yang, J. Lyu, H. Zhou, The WISE detection of an infrared echo in tidal disruption event ASASSN-14li. Astrophys. J. Lett. 828, 14 (2016). https://doi.org/10.3847/2041-8205/ 828/1/L14

N. Jiang, T. Wang, L. Yan, T. Xiao, C. Yang, L. Dou, H. Wang, R. Cutri, A. Mainzer, Mid-infrared flare of TDE candidate PS16dtm: Dust echo and implications for the spectral evolution. Astrophys. J. 850(1), 63 (2017). https://doi.org/10.3847/1538-4357/aa93f5

N. Jiang, T. Wang, G. Mou, H. Liu, L. Dou, Z. Sheng, Y. Wang, Infrared echo and late-stage rebrightening of nuclear transient Ps1-10adi: Exploring the torus with tidal disruption events in active galactic nuclei. Astrophys. J. 871(1), 15 (2019). https://doi.org/10.3847/1538-4357/aaf6b2

N. Jiang, T. Wang, L. Dou, X. Shu, X. Hu, H. Liu, Y. Wang, L. Yan, Z. Sheng, C. Yang, L. Sun, H. Zhou, Mid-InfraRed Outburst in Nearby Galaxies (MIRONG) I: Sample selection and characterization. arXiv e-prints, 2012-06806 (2020). https://doi.org/10.3847/1538-4365/abd1dc

N. Jiang, T. Wang, X. Hu, L. Sun, L. Dou, L. Xiao, Infrared echoes of optical tidal disruption events: $\sim 1 \%$ dust covering factor or less at sub-parsec scale. arXiv e-prints, 2102-08044 (2021). https://doi.org/10. 3847/1538-4357/abe772

E. Kankare, R. Kotak, S. Mattila, P. Lundqvist, M.J. Ward, M. Fraser, A. Lawrence, S.J. Smartt, W.P.S. Meikle, A. Bruce, J. Harmanen, S.J. Hutton, C. Inserra, T. Kangas, A. Pastorello, T. Reynolds, C. Romero-Cañizales, K.W. Smith, S. Valenti, K.C. Chambers, K.W. Hodapp, M.E. Huber, N. Kaiser, R.-P. Kudritzki, E.A. Magnier, J.L. Tonry, R.J. Wainscoat, C. Waters, A population of highly energetic transient events in the centres of active galaxies. Nat. Astron. 1, 865-871 (2017). https://doi.org/10.1038/ s41550-017-0290-2

E. Kara, W.N. Alston, A.C. Fabian, E.M. Cackett, P. Uttley, C.S. Reynolds, A. Zoghbi, A global look at Xray time lags in Seyfert galaxies. Mon. Not. R. Astron. Soc. 462(1), 511-531 (2016a). https://doi.org/ 10.1093/mnras/stw1695

E. Kara, J.M. Miller, C. Reynolds, L. Dai, Relativistic reverberation in the accretion flow of a tidal disruption event. Nature 535(7612), 388-390 (2016b). https://doi.org/10.1038/nature18007

F.P. Keenan, P.H. Norrington, Relative emission line strengths for Fe VII in astrophysical plasmas. Astron. Astrophys. 181, 370-372 (1987). http://adsabs.harvard.edu/abs/1987A\&A...181..370K

S. Komossa, Tidal disruption of stars by supermassive black holes: Status of observations. J. High Energy Astrophys. 7, 148-157 (2015). https://doi.org/10.1016/j.jheap.2015.04.006 
S. Komossa, N. Bade, The giant X-ray outbursts in NGC 5905 and IC 3599: Follow-up observations and outburst scenarios. Astron. Astrophys. 343, 775-787 (1999). http://adsabs.harvard.edu/abs/1999A\%26A... 343..775K

S. Komossa, H. Schulz, Interpretation of the emission line spectra of Seyfert 2 galaxies by multi-component photoionization models. Astron. Astrophys. 323, 31-46 (1997). http://adsabs.harvard.edu/abs/1997A\& A...323...31K

S. Komossa, H. Zhou, T. Wang, M. Ajello, J. Ge, J. Greiner, H. Lu, M. Salvato, R. Saxton, H. Shan, D. $\mathrm{Xu}, \mathrm{W}$. Yuan, Discovery of superstrong, fading, iron line emission and double-peaked Balmer lines of the galaxy SDSS J095209.56+214313.3: The light echo of a huge flare. Astrophys. J. Lett. 678(1), 13 (2008). https://doi.org/10.1086/588281

S. Komossa, H. Zhou, A. Rau, M. Dopita, A. Gal-Yam, J. Greiner, J. Zuther, M. Salvato, D. Xu, H. Lu, R. Saxton, M. Ajello, NTT, Spitzer, and Chandra Spectroscopy of SDSSJ095209.56+214313.3: The most luminous coronal-line supernova ever observed, or a stellar tidal disruption event? Astrophys. J. 701, 105-121 (2009). https://doi.org/10.1088/0004-637X/701/1/105

S. Komossa, The puzzle of SDSSJ0952+2143, in Proceedings of Frontier Objects in Astrophysics and Particle Physics, ed. by F. Giovanelli et al. (2010), p. 10

E.C. Kool, T.M. Reynolds, S. Mattila, E. Kankare, M.A. Pérez-Torres, A. Efstathiou, S. Ryder, C. RomeroCañizales, W. Lu, T. Heikkilä, G.E. Anderson, M. Berton, J. Bright, G. Cannizzaro, D. Eappachen, M. Fraser, M. Gromadzki, P.G. Jonker, H. Kuncarayakti, P. Lundqvist, K. Maeda, R.M. McDermid, A.M. Medling, S. Moran, A. Reguitti, M. Shahband eh, S. Tsygankov, V. U, T. Wevers, AT 2017gbl: A dust obscured TDE candidate in a luminous infrared galaxy. Mon. Not. R. Astron. Soc. 498(2), 2167-2195 (2020). https://doi.org/10.1093/mnras/staa2351

S. Koshida, T. Minezaki, Y. Yoshii, Y. Kobayashi, Y. Sakata, S. Sugawara, K. Enya, M. Suganuma, H. Tomita, T. Aoki, B.A. Peterson, Reverberation measurements of the inner radius of the dust torus in 17 Seyfert galaxies. Astrophys. J. 788, 159 (2014). https://doi.org/10.1088/0004-637X/788/2/159

D. Li, R.D. Saxton, W. Yuan, L. Sun, H.-Y. Liu, N. Jiang, H. Cheng, H. Zhou, S. Komossa, C. Jin, Multiwavelength study of an X-ray tidal disruption event candidate in NGC 5092. Astrophys. J. 891(2), 121 (2020). https://doi.org/10.3847/1538-4357/ab744a

D. Lin, P.W. Maksym, J.A. Irwin, S. Komossa, N.A. Webb, O. Godet, D. Barret, D. Grupe, S.D.J. Gwyn, An ultrasoft X-ray flare from 3XMM J152130.7+074916: A tidal disruption event candidate. Astrophys. J. 811, 43 (2015). https://doi.org/10.1088/0004-637X/811/1/43

Z. Liu, D. Li, H.-Y. Liu, Y. Lu, W. Yuan, L. Dou, R.-F. Shen, A tidal disruption event candidate discovered in the active galactic nucleus SDSS J022700.77-042020.6. Astrophys. J. 894(2), 93 (2020). https://doi. org/10.3847/1538-4357/ab880f

W. Lu, P. Kumar, N.J. Evans, Infrared emission from tidal disruption events - Probing the pc-scale dust content around galactic nuclei. Mon. Not. R. Astron. Soc. 458, 575-581 (2016). https://doi.org/10.1093/mnras/ stw307

W. Lu, J. Krolik, P. Crumley, P. Kumar, Radiative interaction between the relativistic jet and optically thick envelope in tidal disruption events. Mon. Not. R. Astron. Soc. 471(1), 1141-1152 (2017). https://doi. org/10.1093/mnras/stx1668

A. Mainzer, J. Bauer, R.M. Cutri, T. Grav, J. Masiero, R. Beck, P. Clarkson, T. Conrow, J. Dailey, P. Eisenhardt, B. Fabinsky, S. Fajardo-Acosta, J. Fowler, C. Gelino, C. Grillmair, I. Heinrichsen, M. Kendall, J.D. Kirkpatrick, F. Liu, F. Masci, H. McCallon, C.R. Nugent, M. Papin, E. Rice, D. Royer, T. Ryan, P. Sevilla, S. Sonnett, R. Stevenson, D.B. Thompson, S. Wheelock, D. Wiemer, M. Wittman, E. Wright, L. Yan, Initial performance of the NEOWISE reactivation mission. Astrophys. J. 792, 30 (2014). https:// doi.org/10.1088/0004-637X/792/1/30

A. Markowitz, R. Edelson, S. Vaughan, P. Uttley, I.M. George, R.E. Griffiths, S. Kaspi, A. Lawrence, I. McHardy, K. Nandra, K. Pounds, J. Reeves, N. Schurch, R. Warwick, X-ray fluctuation power spectral densities of Seyfert 1 galaxies. Astrophys. J. 593, 96-114 (2003). https://doi.org/10.1086/375330

A.P. Marscher, S.G. Jorstad, K.E. Williamson, A. Lähteenmäki, M. Tornikoski, J.M. Hunter, K.A. Leidig, M. Zain Mobeen, R.J.C. Vera, W. Chamani, X-ray, UV, and radio timing observations of the radio galaxy 3C 120. Astrophys. J. 867, 128 (2018). https://doi.org/10.3847/1538-4357/aae4de

S. Mattila, M. Pérez-Torres, A. Efstathiou, P. Mimica, M. Fraser, E. Kankare, A. Alberdi, M.Á. Aloy, T. Heikkilä, P.G. Jonker, P. Lundqvist, I. Martí-Vidal, W.P.S. Meikle, C. Romero-Cañizales, S.J. Smartt, S. Tsygankov, E. Varenius, A. Alonso-Herrero, M. Bondi, C. Fransson, R. Herrero-Illana, T. Kangas, R. Kotak, N. Ramírez-Olivencia, P. Väisänen, R.J. Beswick, D.L. Clements, R. Greimel, J. Harmanen, J. Kotilainen, K. Nandra, T. Reynolds, S. Ryder, N.A. Walton, K. Wiik, G. Östlin, A dust-enshrouded tidal disruption event with a resolved radio jet in a galaxy merger. Science 361, 482-485 (2018). https://doi. org/10.1126/science.aao4669

J.E. McClintock, R.A. Remillard, in Black Hole Binaries, ed. by W.H.G. Lewin, M. van der Klis (2006), pp. 157-213 
I.M. McHardy, E. Koerding, C. Knigge, P. Uttley, R.P. Fender, Active galactic nuclei as scaled-up Galactic black holes. Nature 444, 730-732 (2006). https://doi.org/10.1038/nature05389

I.M. McHardy, S.D. Connolly, B.M. Peterson, A. Bieryla, H. Chand, M.S. Elvis, D. Emmanoulopoulos, E. Falco, P. Gandhi, S. Kaspi, D. Latham, P. Lira, C. McCully, H. Netzer, M. Uemura, The origin of UVoptical variability in AGN and test of disc models: XMM-Newton and ground-based observations of NGC 4395. Astron. Nachr. 337, 500 (2016). https://doi.org/10.1002/asna.201612337

I.M. McHardy, S.D. Connolly, K. Horne, E.M. Cackett, J. Gelbord, B.M. Peterson, M. Pahari, N. Gehrels, M. Goad, P. Lira, P. Arevalo, R.D. Baldi, N. Brandt, E. Breedt, H. Chand, G. Dewangan, C. Done, M. Elvis, D. Emmanoulopoulos, M.M. Fausnaugh, S. Kaspi, C.S. Kochanek, K. Korista, I.E. Papadakis, A.R. Rao, P. Uttley, M. Vestergaard, M.J. Ward, X-ray/UV/optical variability of NGC 4593 with Swift: Reprocessing of X-rays by an extended reprocessor. Mon. Not. R. Astron. Soc. 480, 2881-2897 (2018). https://doi.org/10.1093/mnras/sty1983

C.W. Morgan, C.S. Kochanek, N.D. Morgan, E.E. Falco, The quasar accretion disk size-black hole mass relation. Astrophys. J. 712, 1129-1136 (2010). https://doi.org/10.1088/0004-637X/712/2/1129

T.J. Moriya, M. Tanaka, T. Morokuma, K. Ohsuga, Superluminous transients at AGN centers from interaction between black hole disk winds and broad-line region clouds. Astrophys. J. Lett. 843(2), 19 (2017). https://doi.org/10.3847/2041-8213/aa7af3

S.E. Motta, T.M. Belloni, L. Stella, T. Muñoz-Darias, R. Fender, Precise mass and spin measurements for a stellar-mass black hole through X-ray timing: The case of GRO J1655-40. Mon. Not. R. Astron. Soc. 437, 2554-2565 (2014). https://doi.org/10.1093/mnras/stt2068

A. Mummery, S.A. Balbus, The spectral evolution of disc dominated tidal disruption events. Mon. Not. R. Astron. Soc. 492(4), 5655-5674 (2020). https://doi.org/10.1093/mnras/staa192

H. Netzer, D. Lutz, M. Schweitzer, A. Contursi, E. Sturm, L.J. Tacconi, S. Veilleux, D.-C. Kim, D. Rupke, A.J. Baker, K. Dasyra, J. Mazzarella, S. Lord, Spitzer Quasar and ULIRG Evolution Study (QUEST). II. The spectral energy distributions of Palomar-Green quasars. Astrophys. J. 666(2), 806-816 (2007). https://doi.org/10.1086/520716

H. Nussbaumer, P.J. Storey, P.J. Storey, Forbidden emission lines of Fe VII. Astron. Astrophys. 113, 21-26 (1982). http://adsabs.harvard.edu/abs/1982A\&A...113...21N

K. O’Brien, K. Horne, R.I. Hynes, W. Chen, C.A. Haswell, M.D. Still, Echoes in X-ray binaries. Mon. Not. R. Astron. Soc. 334, 426-434 (2002). https://doi.org/10.1046/j.1365-8711.2002.05530.x

D.E. Osterbrock, Astrophysics of Gaseous Nebulae and Active Galactic Nuclei (1989)

L. Palaversa, S. Gezari, B. Sesar, J.S. Stuart, P. Wozniak, B. Holl, Ž. Ivezić, Revealing the nature of extreme coronal-line emitter SDSS J095209.56+214313.3. Astrophys. J. 819, 151 (2016). https://doi.org/ 10.3847/0004-637X/819/2/151

D.R. Pasham, S. van Velzen, Discovery of a time lag between the soft X-ray and radio emission of the tidal disruption flare ASASSN-14li: Evidence for linear disk-jet coupling. Astrophys. J. 856(1), 1 (2018). https://doi.org/10.3847/1538-4357/aab361

D.R. Pasham, S.B. Cenko, A. Sadowski, J. Guillochon, N.C. Stone, S. van Velzen, J.K. Cannizzo, Optical/UV-to-X-ray echoes from the tidal disruption flare ASASSN-14li. Astrophys. J. Lett. 837, 30 (2017). https://doi.org/10.3847/2041-8213/aa6003

D.R. Pasham, R.A. Remillard, P.C. Fragile, A. Franchini, N.C. Stone, G. Lodato, J. Homan, D. Chakrabarty, F.K. Baganoff, J.F. Steiner, E.R. Coughlin, N.R. Pasham, A loud quasi-periodic oscillation after a star is disrupted by a massive black hole. Science 363(6426), 531-534 (2019). https://doi.org/10.1126/science. aar7480

B.M. Peterson, L. Ferrarese, K.M. Gilbert, S. Kaspi, M.A. Malkan, D. Maoz, D. Merritt, H. Netzer, C.A. Onken, R.W. Pogge, Central masses and broad-line region sizes of active galactic nuclei. II. A homogeneous analysis of a large reverberation-mapping database. Astrophys. J. 613(2), 682-699 (2004). https:// doi.org/10.1086/423269

T. Piran, G. Svirski, J. Krolik, R.M. Cheng, H. Shiokawa, Disk formation versus disk accretion - What powers tidal disruption events? Astrophys. J. 806, 164 (2015). https://doi.org/10.1088/0004-637X/806/2/164

J.C. Raymond, W.P. Blair, K.S. Long, Detection of ultraviolet emission lines in SN 1006 with the Hopkins ultraviolet telescope. Astrophys. J. Lett. 454, 31 (1995). https://doi.org/10.1086/309772

R.C. Reis, J.M. Miller, M.T. Reynolds, K. Gültekin, D. Maitra, A.L. King, T.E. Strohmayer, A 200-second quasi-periodicity after the tidal disruption of a star by a dormant black hole. Science 337, 949 (2012). https://doi.org/10.1126/science.1223940

R.A. Remillard, G.J. Sobczak, M.P. Muno, J.E. McClintock, Characterizing the quasi-periodic oscillation behavior of the X-ray nova XTE J1550-564. Astrophys. J. 564, 962-973 (2002). https://doi.org/10. $1086 / 324276$

C.S. Reynolds, M.A. Nowak, Fluorescent iron lines as a probe of astrophysical black hole systems. Phys. Rep. 377, 389-466 (2003). https://doi.org/10.1016/S0370-1573(02)00584-7 
C. Romero-Cañizales, S. Mattila, A. Alberdi, M.A. Pérez-Torres, E. Kankare, S.D. Ryder, The core-collapse supernova rate in Arp 299 revisited. Mon. Not. R. Astron. Soc. 415(3), 2688-2698 (2011). https://doi. org/10.1111/j.1365-2966.2011.18886.x

N. Roth, E.M. Rossi, J. Krolik, T. Piran, B. Mockler, D. Kasen, Radiative emission mechanisms. Space Sci. Rev. 216(7), 114 (2020). https://doi.org/10.1007/s11214-020-00735-1

N.I. Shakura, R.A. Sunyaev, Black holes in binary systems. Observational appearance. Astron. Astrophys. 24, 337-355 (1973). http://adsabs.harvard.edu/abs/1973A\%26A...24..337S

R. Stein, S. van Velzen, M. Kowalski, A. Franckowiak, S. Gezari, J.C.A. Miller-Jones, S. Frederick, I. Sfaradi, M.F. Bietenholz, A. Horesh, R. Fender, S. Garrappa, T. Ahumada, I. Andreoni, J. Belicki, E.C. Bellm, M. Böttcher, V. Brinnel, R. Burruss, S.B. Cenko, M.W. Coughlin, V. Cunningham, A. Drake, G.R. Farrar, M. Feeney, R.J. Foley, A. Gal-Yam, V.Z. Golkhou, A. Goobar, M.J. Graham, E. Hammerstein, G. Helou, T. Hung, M.M. Kasliwal, C.D. Kilpatrick, A.K.H. Kong, T. Kupfer, R.R. Laher, A.A. Mahabal, F.J. Masci, J. Necker, J. Nordin, D.A. Perley, M. Rigault, S. Reusch, H. Rodriguez, C. Rojas-Bravo, B. Rusholme, D.L. Shupe, L.P. Singer, J. Sollerman, M.T. Soumagnac, D. Stern, K. Taggart, J. van Santen, C. Ward, P. Woudt, Y. Yao, A high-energy neutrino coincident with a tidal disruption event. arXiv e-prints, 2005-05340 (2020). https://doi.org/10.1038/s41550-020-01295-8

N.C. Stone, A. Generozov, E. Vasiliev, B.D. Metzger, The delay time distribution of tidal disruption flares. Mon. Not. R. Astron. Soc. 480, 5060-5077 (2018). https://doi.org/10.1093/mnras/sty2045

L. Sun, N. Jiang, T. Wang, H. Zhou, L. Dou, C. Yang, X. Pan, Z. Sheng, Z. Zhong, L. Yan, G. Li, A midinfrared flare in the active galaxy MCG-02-04-026: Dust echo of a nuclear transient event. Astrophys. J. 898(2), 129 (2020). https://doi.org/10.3847/1538-4357/ab9f2c

C. Tadhunter, R. Spence, M. Rose, J. Mullaney, P. Crowther, A tidal disruption event in the nearby ultraluminous infrared galaxy F01004-2237. Nat. Astron. 1, 0061 (2017). https://doi.org/10.1038/s41550017-0061

C. Taylor, C.S. Reynolds, Exploring the effects of disk thickness on the black hole reflection spectrum. Astrophys. J. 855(2), 120 (2018). https://doi.org/10.3847/1538-4357/aaad63

L.L. Thomsen, J. Lixin Dai, E. Ramirez-Ruiz, E. Kara, C. Reynolds, X-ray fluorescence from superEddington accreting black holes. Astrophys. J. Lett. 884(1), 21 (2019). https://doi.org/10.3847/2041$8213 / \mathrm{ab} 4518$

P. Uttley, E.M. Cackett, A.C. Fabian, E. Kara, D.R. Wilkins, X-ray reverberation around accreting black holes. Astron. Astrophys. Rev. 22, 72 (2014). https://doi.org/10.1007/s00159-014-0072-0

S. van Velzen, V. Gorjian, J. Krolik, A. Mendez, Dust reprocessing of stellar tidal disruption flares. Spitzer Proposal (2015). http://adsabs.harvard.edu/abs/2015sptz.prop12046V

S. van Velzen, A.J. Mendez, J.H. Krolik, V. Gorjian, Discovery of transient infrared emission from dust heated by stellar tidal disruption flares. Astrophys. J. 829, 19 (2016). https://doi.org/10.3847/0004-637X/829/ $1 / 19$

S. van Velzen, G.C. Bower, B.D. Metzger, Science with an ngVLA: Tidal disruption events, in Science with a Next Generation Very Large Array, ed. by E. Murphy. Astron. Soc. Pac. Conference Series, vol. 517 (2018), p. 737. http://adsabs.harvard.edu/abs/2018ASPC..517..737V

S. van Velzen, N.C. Stone, B.D. Metzger, S. Gezari, T.M. Brown, A.S. Fruchter, Late-time UV observations of tidal disruption flares reveal unobscured, compact accretion disks. Astrophys. J. 878(2), 82 (2019). https://doi.org/10.3847/1538-4357/ab1844

S. van Velzen, T.W.-S. Holoien, F. Onori, T. Hung, I. Arcavi, Optical-ultraviolet tidal disruption events. Space Sci. Rev. 216(8), 124 (2020). https://doi.org/10.1007/s11214-020-00753-z

S. van Velzen, S. Gezari, E. Hammerstein, N. Roth, S. Frederick, C. Ward, T. Hung, S.B. Cenko, R. Stein, D.A. Perley, K. Taggart, R.J. Foley, J. Sollerman, N. Blagorodnova, I. Andreoni, E.C. Bellm, V. Brinnel, K. De, R. Dekany, M. Feeney, C. Fremling, M. Giomi, V.Z. Golkhou, M.J. Graham, A.Y.Q. Ho, M.M. Kasliwal, C.D. Kilpatrick, S.R. Kulkarni, T. Kupfer, R.R. Laher, A. Mahabal, F.J. Masci, A.A. Miller, J. Nordin, R. Riddle, B. Rusholme, J. van Santen, Y. Sharma, D.L. Shupe, M.T. Soumagnac, Seventeen Tidal Disruption Events from the First Half of ZTF Survey Observations: Entering a New Era of Population Studies. Astrophys. J. 908(1), 4 (2021). https://doi.org/10.3847/1538-4357/abc258

S. Vaughan, P. Uttley, Where are the X-ray quasi-periodic oscillations in active galaxies? Mon. Not. R. Astron. Soc. 362, 235-244 (2005). https://doi.org/10.1111/j.1365-2966.2005.09296.X

T.-G. Wang, H.-Y. Zhou, L.-F. Wang, H.-L. Lu, D. Xu, Transient superstrong coronal lines and broad bumps in the galaxy SDSS J074820.67+471214.3. Astrophys. J. 740(2), 85 (2011). https://doi.org/10.1088/ 0004-637X/740/2/85

T.-G. Wang, H.-Y. Zhou, S. Komossa, H.-Y. Wang, W. Yuan, C. Yang, Extreme coronal line emitters: Tidal disruption of stars by massive black holes in galactic nuclei? Astrophys. J. 749, 115 (2012). https://doi. org/10.1088/0004-637X/749/2/115

T. Wang, L. Yan, L. Dou, N. Jiang, Z. Sheng, C. Yang, Long-term decline of the mid-infrared emission of normal galaxies: Dust echo of tidal disruption flare? Mon. Not. R. Astron. Soc. 477, 2943-2965 (2018). https://doi.org/10.1093/mnras/sty465 
E. Waxman, B.T. Draine, Dust sublimation by gamma-ray bursts and its implications. Astrophys. J. 537, 796-802 (2000). https://doi.org/10.1086/309053

J.C. Weingartner, B.T. Draine, Dust grain-size distributions and extinction in the Milky Way, Large Magellanic Cloud, and Small Magellanic Cloud. Astrophys. J. 548, 296-309 (2001). https://doi.org/10.1086/ 318651

T. Wevers, D.R. Pasham, S. van Velzen, G. Leloudas, S. Schulze, J.C.A. Miller-Jones, P.G. Jonker, M. Gromadzki, E. Kankare, S.T. Hodgkin, Ł. Wyrzykowski, Z. Kostrzewa-Rutkowska, S. Moran, M. Berton, K. Maguire, F. Onori, S. Mattila, M. Nicholl, Evidence for rapid disc formation and reprocessing in the X-ray bright tidal disruption event candidate AT 2018fyk. Mon. Not. R. Astron. Soc. 488(4), 4816-4830 (2019). https://doi.org/10.1093/mnras/stz1976

T. Wevers, D.R. Pasham, S. van Velzen, J.C.A. Miller-Jones, P. Uttley, K. Gendreau, R. Remillard, Z. Arzoumanian, M. Loewenstein, A. Chiti, Rapid accretion state transitions following the tidal disruption event AT2018fyk. arXiv e-prints, 2101-04692 (2021). https://doi.org/10.3847/1538-4357/abf5e2

R.J. White, B.M. Peterson, Comments on cross-correlation methodology in variability studies of active galactic nuclei. Publ. Astron. Soc. Pac. 106, 879 (1994). https://doi.org/10.1086/133456

D.R. Wilkins, E.M. Cackett, A.C. Fabian, C.S. Reynolds, Towards modelling X-ray reverberation in AGN: Piecing together the extended corona. Mon. Not. R. Astron. Soc. 458(1), 200-225 (2016). https://doi. org/10.1093/mnras/stw276

E.L. Wright, P.R.M. Eisenhardt, A.K. Mainzer, M.E. Ressler, R.M. Cutri, T. Jarrett, J.D. Kirkpatrick, D. Padgett, R.S. McMillan, M. Skrutskie, S.A. Stanford, M. Cohen, R.G. Walker, J.C. Mather, D. Leisawitz, T.N. Gautier III, I. McLean, D. Benford, C.J. Lonsdale, A. Blain, B. Mendez, W.R. Irace, V. Duval, F. Liu, D. Royer, I. Heinrichsen, J. Howard, M. Shannon, M. Kendall, A.L. Walsh, M. Larsen, J.G. Cardon, S. Schick, M. Schwalm, M. Abid, B. Fabinsky, L. Naes, C.-W. Tsai, The Wide-field Infrared Survey Explorer (WISE): Mission description and initial on-orbit performance. Astron. J. 140, 1868-1881 (2010). https://doi.org/10.1088/0004-6256/140/6/1868

L. Yan, T. Wang, N. Jiang, D. Stern, L. Dou, C. Fremling, M.J. Graham, A.J. Drake, C. Yang, K. Burdge, M.M. Kasliwal, Rapid turn-on of type-1 AGN in a quiescent early-type galaxy SDSS1115+0544. Astrophys. J. 874(1), 44 (2019). https://doi.org/10.3847/1538-4357/ab074b

C.-W. Yang, T.-G. Wang, G. Ferland, W. Yuan, H.-Y. Zhou, P. Jiang, Long-term spectral evolution of tidal disruption candidates selected by strong coronal lines. Astrophys. J. 774, 46 (2013). https://doi.org/10. 1088/0004-637X/774/1/46

Q. Yang, Y. Shen, X. Liu, X.-B. Wu, L. Jiang, J. Shangguan, M.J. Graham, S. Yao, An unusual mid-infrared flare in a type 2 AGN: An obscured turning-on AGN or tidal disruption event? Astrophys. J. 885(2), 110 (2019). https://doi.org/10.3847/1538-4357/ab481a

W. Yuan, C. Zhang, H. Feng, S.N. Zhang, Z.X. Ling, D. Zhao, J. Deng, Y. Qiu, J.P. Osborne, P. O'Brien, R. Willingale, J. Lapington, G.W. Fraser, the Einstein Probe team, Einstein Probe - A small mission to monitor and explore the dynamic X-ray Universe. arXiv e-prints, 1506-07735 (2015)

A. Zabludoff, I. Arcavi, S. La Massa, H.B. Perets, B. Trakhtenbrot, B.A. Zauderer, K. Auchettl, J.L. Dai, K.D. French, T. Hung, E. Kara, G. Lodato, W.P. Maksym, Y. Qin, E. Ramirez-Ruiz, N. Roth, J.C. Runnoe, T. Wevers, Distinguishing tidal disruption events from impostors. arXiv e-prints, 2103-12150 (2021). https://doi.org/10.1007/s11214-021-00829-4

B.A. Zauderer, E. Berger, A.M. Soderberg, A. Loeb, R. Narayan, D.A. Frail, G.R. Petitpas, A. Brunthaler, R. Chornock, J.M. Carpenter, G.G. Pooley, K. Mooley, S.R. Kulkarni, R. Margutti, D.B. Fox, E. Nakar, N.A. Patel, N.H. Volgenau, T.L. Culverhouse, M.F. Bietenholz, M.P. Rupen, W. Max-Moerbeck, A.C.S. Readhead, J. Richards, M. Shepherd, S. Storm, C.L.H. Hull, Birth of a relativistic outflow in the unusual $\gamma$-ray transient Swift J164449.3+573451. Nature 476, 425-428 (2011). https://doi.org/10.1038/ nature 10366

A. Zoghbi, A.C. Fabian, C.S. Reynolds, E.M. Cackett, Relativistic iron K X-ray reverberation in NGC 4151. Mon. Not. R. Astron. Soc. 422(1), 129-134 (2012). https://doi.org/10.1111/j.1365-2966.2012.20587.x

Publisher's Note Springer Nature remains neutral with regard to jurisdictional claims in published maps and institutional affiliations. 